\title{
Surviving the hole
}

\section{Spatially resolved chemistry around Sagittarius $A^{* \star}$}

\author{
S. Martín ${ }^{1,2}$, J. Martín-Pintado ${ }^{3}$, M. Montero-Castaño ${ }^{4}$, P. T. P. $\mathrm{Ho}^{2,5}$, and R. Blundell ${ }^{2}$ \\ ${ }^{1}$ European Southern Observatory, Alonso de Córdova 3107, Vitacura, Casilla 19001, Santiago 19, Chile \\ e-mail: smartin@eso.org \\ 2 Harvard-Smithsonian Center for Astrophysics, 60 Garden St., 02138, Cambridge, MA, USA \\ 3 Centro de Astrobiología (CSIC-INTA), Ctra. de Torrejón Ajalvir, km 4, 28850 Torrejón de Ardoz, Madrid, Spain \\ ${ }^{4}$ Department of Astronomy, Columbia University, 550 West 120th Street, New York, NY 10027, USA \\ 5 Institute of Astronomy and Astrophysics, Academia Sinica, PO Box 23141, Taipei 106, Taiwan
}

Received 17 May 2011 / Accepted 1 December 2011

\begin{abstract}
Context. The interstellar region within the few central parsecs around the super-massive black hole, Sgr A*, at the very Galactic center is composed of a number of overlapping molecular structures that are subject to one of the most hostile physical environments in the Galaxy.

Aims. Through the study of the morphology and kinematics of the emission from different molecular species as well as the variation of their line ratios we can attain a deeper understanding of the distribution and interaction between different gas structures and the energetic phenomena taking place in the surroundings of Sgr $\mathrm{A}^{*}$ and of the physical processes responsible for the heating in this region.

Methods. We performed high-resolution ( $\left.4^{\prime \prime} \times 3^{\prime \prime} \sim 0.16 \times 0.11 \mathrm{pc}\right)$ interferometric observations of $\mathrm{CN},{ }^{13} \mathrm{CN}, \mathrm{H}_{2} \mathrm{CO}, \mathrm{SiO}, \mathrm{c}-\mathrm{C}_{3} \mathrm{H}_{2}$ and $\mathrm{HC}_{3} \mathrm{~N}$ emission at $1.3 \mathrm{~mm}$ toward the central $\sim 4 \mathrm{pc}$ of the Galactic center region.

Results. Strong differences are observed in the distribution of the different molecules. The UV resistant species CN, the only species tracing all previously identified circumnuclear disk (CND) structures, is mostly concentrated in optically thick clumps in the rotating filaments around $\mathrm{Sgr} \mathrm{A}^{*} . \mathrm{H}_{2} \mathrm{CO}$ emission traces a shell-like structure that we interpret as the expansion of Sgr A East against the $50 \mathrm{~km} \mathrm{~s}^{-1}$ and $20 \mathrm{~km} \mathrm{~s}^{-1}$ giant molecular clouds (GMCs). We derive isotopic ratios ${ }^{12} \mathrm{C} /{ }^{13} \mathrm{C} \sim 15-45$ across most of the CND region, except for the northeast arm, where the peak of $\mathrm{H}_{2} \mathrm{CO}$ is observed and ratios $<10$ are found. The densest molecular material, traced by $\mathrm{SiO}$ and $\mathrm{HC}_{3} \mathrm{~N}$, is located in the southern $\mathrm{CND}$, likely because of shocked gas infalling from the $20 \mathrm{~km} \mathrm{~s}^{-1} \mathrm{GMC}$ streamers, and the northeast arm, as a result of the expansion of Sgr A East or a connecting point of the $50 \mathrm{~km} \mathrm{~s}^{-1}$ streamer east of the CND. The observed $\mathrm{c}-\mathrm{C}_{3} \mathrm{H}_{2} / \mathrm{HC}_{3} \mathrm{~N}$ ratio observed in the region is more than an order of magnitude lower than in Galactic PDRs. Toward the central region only $\mathrm{CN}$ was detected in absorption. In addition to the known narrow line-of-sight absorptions, a $90 \mathrm{~km} \mathrm{~s}^{-1}$ wide optically thick spectral feature is observed. We found evidence of an even wider $\left(>100 \mathrm{~km} \mathrm{~s}^{-1}\right)$ absorption feature. About $70-75 \%$ of the gas mass, concentrated in just the $27 \%$ densest molecular clumps, is associated with rotating structures and show evidence of an association with each of the arcs of ionized gas in the mini-spiral structure.

Conclusions. These observations provide a combined chemical and kinematical picture of the very central region around Sgr A* Chemical differentiation has been proven to be a powerful tool to distinguish the many overlapping molecular components in this crowded and heavily obscured region.
\end{abstract}

Key words. ISM: molecules - ISM: clouds - radio lines: ISM - Galaxy: center - ISM: kinematics and dynamics

\section{Introduction}

At a distance of $\sim 8.0 \mathrm{kpc}$ (Reid 1993; Groenewegen et al. 2008; Ghez et al. 2008) the few central parsecs around the super-massive black hole at the dynamic center of the Galaxy $\left(4.1 \pm 0.6 \times 10^{6} M_{\odot}\right.$, Ghez et al. 2008) are likely one of the most hostile environments for the interstellar medium (ISM) in the Milky Way. In this region the molecular gas undergoes strong disruptive tidal forces and pervasive X-ray irradiation $\left(L_{X}(20-120 \mathrm{keV}) \sim 2.6 \times 10^{35} \mathrm{erg} \mathrm{s}^{-1}\right.$; Bélanger et al. 2006) in the vicinity of the black hole. Likewise, the ISM is subject to supernova remnants (SNR), HII regions, and strong photodissociating UV fields from the central cluster $\left(L \sim 1-3 \times 10^{7} L_{\odot}\right.$ and

^ Figures 12-22 and Tables 3 and 4 are available in electronic form at http://www . aanda.org ionizing photon flux of $2 \times 10^{50} \mathrm{~s}^{-1}$; Lacy et al. 1980; Davidson et al. 1992).

The super-massive black hole is associated with the compact strong non thermal source $\mathrm{Sgr} \mathrm{A}^{*}$. The mini-spiral of ionized gas consisting of three streamers known as Sgr A West (Lo \& Claussen 1983; Ekers et al. 1983) is likely feeding the central source. Surrounding Sgr A West, the so-called circumnuclear disk (CND) is a clumpy ringlike structure rotating counterclockwise around the dynamical center (Guesten et al. 1987; Wright et al. 2001; Christopher et al. 2005). The molecular ring extends from its inner sharp edge at a projected distance of $1.6 \mathrm{pc}$ from Sgr A*, out to a smoother edge at $\sim 2$ pc (Christopher et al. 2005). However, the CND appears not to be a single symmetric structure forming a coherent ring (Marshall et al. 1995). It has been suggested that the CND is a warped structure (Guesten et al. 1987) or, because of the incompleteness of the ring, formed by 
distinct rotating structures (Jackson et al. 1993; Wright et al. 2001). Most of the observed molecular clumps forming the CND, with diameters of $\sim 7^{\prime \prime}$, are tidally stable and in virial equilibrium (Christopher et al. 2005). Thus, the rotating molecular structures composing the CND appear to be non transient structures with an estimated lifetime of $\sim 10^{7} \mathrm{yr}$ (Christopher et al. 2005; Montero-Castaño et al. 2009).

A number of molecular emission observations have been carried out to study the CND structure, its relation to the Sgr A West streamers, and the possible interaction with the two giant molecular clouds (GMC), namely the $20 \mathrm{~km} \mathrm{~s}^{-1}$ and $50 \mathrm{~km} \mathrm{~s}^{-1}$ GMCs (see Ho et al. 1991; Marr et al. 1993; Montero-Castaño et al. 2009, for references). So far, the only species with highresolution aperture synthesis observations are the $\mathrm{HCN} 1-0$ (Guesten et al. 1987; Wright et al. 2001; Christopher et al. 2005), 3-2 (Marshall et al. 1995), and 4-3 (Marshall et al. 1995; Montero-Castaño et al. 2009), the $1-0$ transitions of $\mathrm{H}^{13} \mathrm{CN}$ and $\mathrm{HCO}^{+}$(Marr et al. 1993; Wright et al. 2001; Christopher et al. 2005), the CS 7-6 (Montero-Castaño et al. 2009), and $\mathrm{NH}_{3}(1,1),(2,2),(3,3),(6,6)$ (McGary et al. 2001; Herrnstein $\&$ Ho 2005). The emission from the high-density tracers like $\mathrm{HCN}, \mathrm{HCO}^{+}$, and $\mathrm{CN}$ nicely trace all molecular features of the $\mathrm{CND}$, though significant differences are observed in the line ratios. These differences are attributed to both abundance and density variations across the $\mathrm{CND}$ (e.g. $\mathrm{HCO}^{+} / \mathrm{HCN}$, Christopher et al. 2005). Although also tracing the CND, the emission of $\mathrm{NH}_{3}$ significantly differs from the emission of the other tracers. The recent work at low resolution by Amo-Baladrón et al. (2011) studies, among other tracers, the emission of $\mathrm{SiO}$ and HNCO in the central 12 pc around Sgr A*. These species show strong abundance variations caused by the influence of shocks and UV radiation in this region (Martín et al. 2008). They probe the potential of $\mathrm{HNCO} / \mathrm{SiO}$ abundance ratio as a measurement of the distance to the central cluster, which provides an insight in the 3-D arrangement of the different molecular components.

In this paper we report the high-resolution $\left(4^{\prime \prime} \times 3^{\prime \prime} \sim\right.$ $0.16 \times 0.11 \mathrm{pc}$ ) observations of $\mathrm{CN},{ }^{13} \mathrm{CN}, \mathrm{H}_{2} \mathrm{CO}, \mathrm{SiO}, \mathrm{c}-\mathrm{C}_{3} \mathrm{H}_{2}$, and $\mathrm{HC}_{3} \mathrm{~N}$. As illustrated by the three-color composite image shown in Fig. 1, each molecular species traces different molecular components as a result of the varying physical and chemical conditions in the vicinity of Sgr $\mathrm{A}^{*}$.

\section{Observations}

Observations were carried out with the Submillimeter Array ${ }^{1}$ (SMA, Ho et al. 2004) in Mauna Kea, Hawaii. We tuned the $230 \mathrm{GHz}$ receivers to observe two spectral windows centered at the frequencies of $217.41 \mathrm{GHz}$ (LSB) and $227.38 \mathrm{GHz}$ (USB). Correlator configuration provided $2 \times 1.968 \mathrm{GHz}$ wide bands at a $0.8125 \mathrm{MHz}$ spectral resolution.

A total of three SMA observing tracks were acquired in its compact and subcompact configurations. Specific observing and calibration details on each of the three SMA tracks are summarized in Table 1. Weather conditions were good (PWV $\sim 3 \mathrm{~mm}$ ) during compact observations and only mediocre (PWV 4-5 mm) during the subcompact. However, phase was observed to be stable in all three tracks.

The observations in the subcompact configuration data were the key to recover the extended emission from the

\footnotetext{
1 The Submillimeter Array is a joint project between the Smithsonian Astrophysical Observatory and the Academia Sinica Institute of Astronomy and Astrophysics and is funded by the Smithsonian Institution and the Academia Sinica.
}

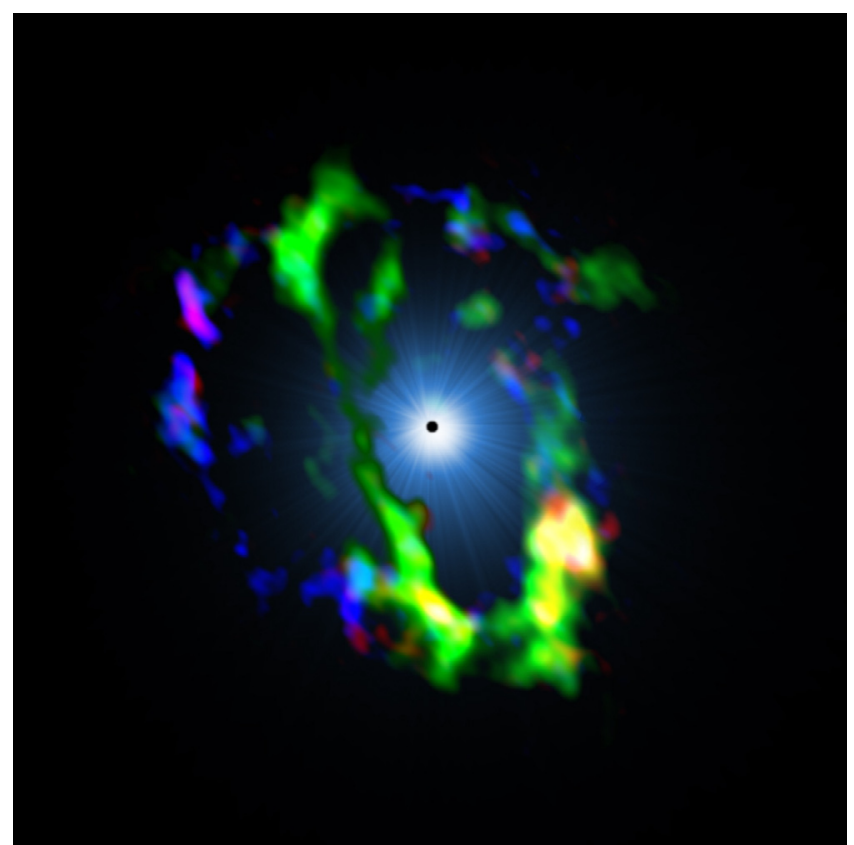

Fig. 1. Three-color composite image of the CND around Sgr A* including the emission of $\mathrm{SiO}$ (red), $\mathrm{CN}$ (green), $\mathrm{H}_{2} \mathrm{CO}$ (blue). The central glow shows an artistic representation of the pervasive X-ray and UVfields from the black hole and central star cluster. In the center, the black hole position is illustrated with a size corresponding to the map resolution.

Table 1. Observational details of each frequency setup.

\begin{tabular}{|c|c|c|c|c|c|}
\hline \multirow[t]{2}{*}{ Date } & \multirow{2}{*}{$\begin{array}{l}\text { Config. }{ }^{a} \\
\text { (\# ant.) }\end{array}$} & \multirow[t]{2}{*}{$\tau_{225^{b}}$} & \multicolumn{3}{|c|}{ Calibrators } \\
\hline & & & Flux & Gain & Bandpass \\
\hline 2007 Jul. 10 & $\mathrm{C}(8)$ & 0.18 & Uranus & $\begin{array}{c}\text { Callisto } \\
\text { J1924-292 }\end{array}$ & $3 \mathrm{c} 454.3$ \\
\hline 2007 Jul. 15 & $\mathrm{C}(8)$ & 0.16 & Uranus & $\begin{array}{c}\text { Callisto } \\
\text { J1924-292 }\end{array}$ & $3 \mathrm{c} 454.3$ \\
\hline 2008 Sep. 03 & $\mathrm{SC}(7)$ & 0.25 & Callisto & $\begin{array}{l}\mathrm{J} 1733-130 \\
\mathrm{~J} 1924-292\end{array}$ & $3 c 454.3$ \\
\hline
\end{tabular}

Notes. ${ }^{(a)}$ Array configuration (SC: Subcompact - C: Compact) and number of antennae available. ${ }^{(b)}$ Average zenith opacity at $225 \mathrm{GHz}$ during observations.

CND structure. This flux recovery is perfectly illustrated by comparing the observations of $\mathrm{HCN}$ 4-3 with just the compact configuration data reported by Montero-Castaño et al. (2006) and the final data set including the subcompact configuration data (Montero-Castaño et al. 2009). The combination of both configurations resulted in projected baselines between $8 \mathrm{~m}(6 \mathrm{k} \lambda)$ and $135 \mathrm{~m}(90 \mathrm{k} \lambda)$, which is equivalent to structure sizes between $34^{\prime \prime}$ and $2^{\prime \prime}$, respectively. Nevertheless, the final cleaned images show negative flux areas nearby the brightest emission regions, which are indicative of the significant missing extended $\left(>30^{\prime \prime}\right)$ emission.

We observed a 9-pointing mosaic centered at $\alpha_{\mathrm{J} 2000}=$ $17^{\mathrm{h}} 45^{\mathrm{m}} 40^{\mathrm{s}}, \delta_{\mathrm{J} 2000}=-29^{\circ} 00^{\prime} 28^{\prime \prime}$ and arranged with a $26^{\prime \prime}$ spacing and a declination offset between each 3-point column of 13" as shown in Fig. 2. This mosaic configuration was aimed to fully sample the elongated CND structure and all features identified in the high-resolution maps of HCN 4-3 by Montero-Castaño et al. (2009). The observation was performed in short 4-min integrations on each of the mosaic pointings with 3-min integrations on the gain calibrators every $\sim 20 \mathrm{~min}$. The combination of the 


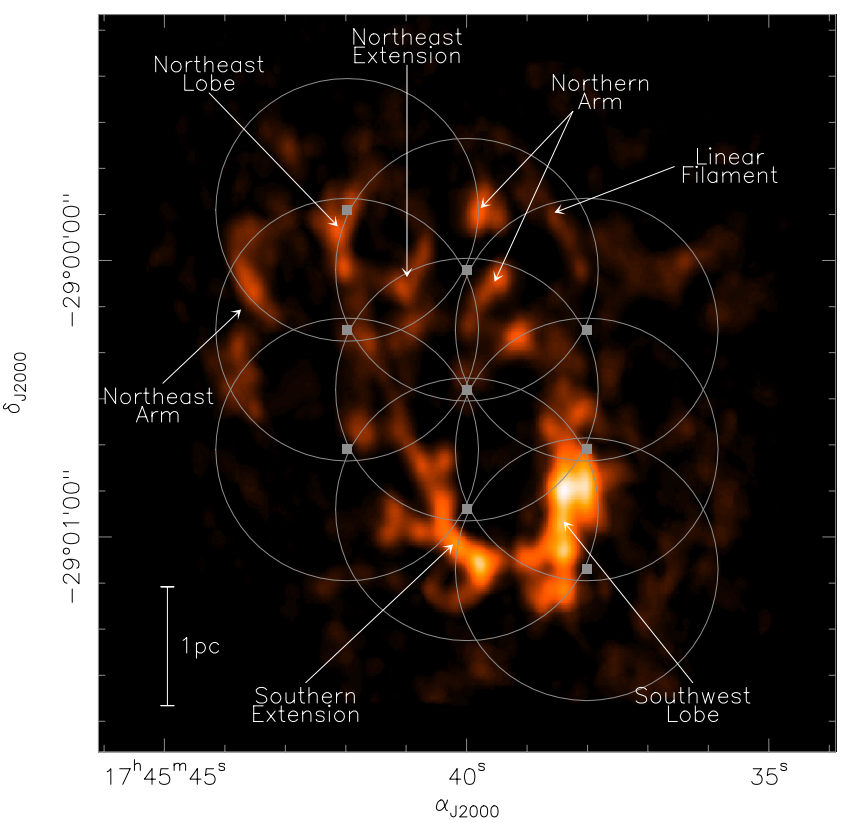

Fig. 2. Central position and primary beam of each of the 9-field mosaic observations. Background image is the HCN 4-3 emission by Montero-Castaño et al. (2009). The different molecular structures labels are adopted from Christopher et al. (2005) and Montero-Castaño et al. (2009).

three tracks resulted in a total integration time per pointing of $\sim 84$ min.

Continuum subtraction was performed in the UV plane using the available line-free channels between all identified molecular features. Data calibration and reduction were performed using the MIR-IDL package, while we used MIRIAD (Sault et al. 1995) for cleaning and GILDAS ${ }^{2}$ for data display and analysis. The natural weighting of the visibilities resulted in a beam size of $4.1^{\prime \prime} \times 2.9^{\prime \prime}$ with a position angle of $\sim 30^{\circ}$. The theoretical rms noise for individual pointings (assuming no overlapping) was $\sim 40 \mathrm{mJy} \mathrm{beam}^{-1}$ in $10 \mathrm{~km} \mathrm{~s}^{-1}$ channels. The final achieved rms noise was $\sim 31$ and $34 \mathrm{mJy}^{-1}$ beam $^{-1}$ for the LSB and USB, respectively, as measured in the central $40^{\prime \prime}$ region in line free channels.

\section{Results}

Within the total $\sim 4 \mathrm{GHz}$ frequency band observed, we have detected the emission of six molecular species. Figure 3 shows the sample spectra extracted from the resulting data cubes at the position of the brightest molecular emission (position 1 in Fig. 4) where the entire identified spectral features are labeled. Basic spectroscopic details for each molecular transition are given in Table 2. Additionally, the emission of $\mathrm{HC}_{3} \mathrm{~N} J=24-23$ emission at $218.3 \mathrm{GHz}$ is detected close to the $\mathrm{H}_{2} \mathrm{CO}$ transition. However, this line is truncated at the lower velocities and therefore was not included in this work.

In Fig. 4 we present the integrated intensity maps from each of the detected transitions. The emission of $\mathrm{SiO}, \mathrm{C}_{3} \mathrm{H}_{2}$, and $\mathrm{HC}_{3} \mathrm{~N}$ was integrated in the velocity range between $-140 \mathrm{~km} \mathrm{~s}^{-1}$ and $+140 \mathrm{~km} \mathrm{~s}^{-1}$ where emission is detected. We integrated between $-190 \mathrm{~km} \mathrm{~s}^{-1}$ and $+470 \mathrm{~km} \mathrm{~s}^{-1}$ for CN so that the two groups of transitions are included in the image. Similarly, ${ }^{13} \mathrm{CN}$ emission was integrated from $-190 \mathrm{~km} \mathrm{~s}^{-1}$ up to

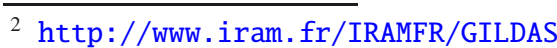
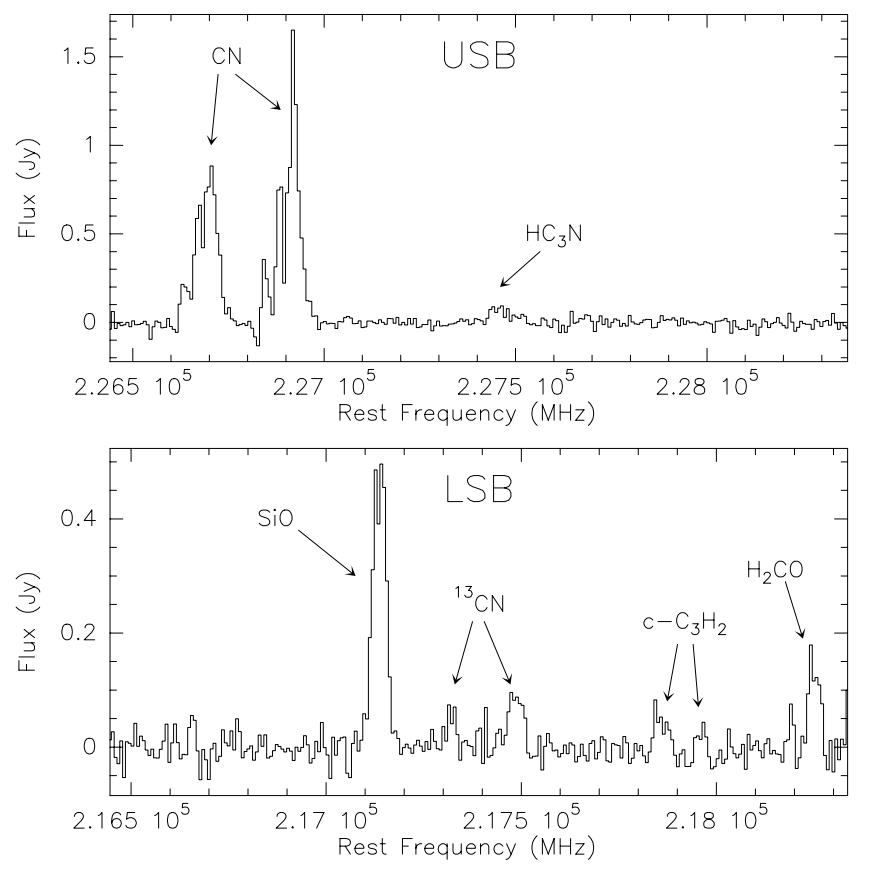

Fig. 3. Sample spectra extracted from a 4 " size square region at the position 1 indicated in Fig. 4. The spectra of both USB and LSB are shown with the identified spectral transitions labeled.

Table 2. Spectral parameters of the transitions detected.

\begin{tabular}{lcccc}
\hline \hline Molecule & Transition & $\begin{array}{c}\text { Frequency } \\
(\mathrm{GHz})\end{array}$ & $\begin{array}{c}E_{\text {upper }}{ }^{a} \\
(\mathrm{~K})\end{array}$ & $\begin{array}{c}A_{\text {ull }}{ }^{2} \\
\left(10^{-4} \mathrm{~s}^{-1}\right)\end{array}$ \\
\hline $\mathrm{SiO}$ & $5-4$ & 217.104 & 31 & 5.2 \\
${ }^{13} \mathrm{CN}^{c}$ & $2-1$ & 217.467 & 16 & 1.0 \\
$\mathrm{c}^{-} \mathrm{C}_{3} \mathrm{H}_{2}$ & $6_{1,6}-5_{0,5}$ & 217.822 & 39 & 5.9 \\
$\mathrm{H}_{2} \mathrm{CO}$ & $3_{0,3}-2_{0,2}$ & 218.222 & 21 & 2.8 \\
$\mathrm{CN}^{c}$ & $2-1$ & 226.875 & 16 & 1.1 \\
$\mathrm{HC}_{3} \mathrm{~N}$ & $25-24$ & 227.419 & 142 & 9.3 \\
\hline
\end{tabular}

Notes. ${ }^{(a)}$ Energy of the transition upper level. ${ }^{(b)}$ Einstein coefficient. (c) Frequency and $A_{\mathrm{ul}}$ refer to the brightest transition of the hyperfine group.

$+350 \mathrm{~km} \mathrm{~s}^{-1}$, where $\mathrm{SiO}$ starts showing emission. Finally, $\mathrm{H}_{2} \mathrm{CO}$ was integrated between $-80 \mathrm{~km} \mathrm{~s}^{-1}$ and $+140 \mathrm{~km} \mathrm{~s}^{-1}$ to avoid the significant emission from $\mathrm{HC}_{3} \mathrm{~N} 24-23$ (at $218.324 \mathrm{GHz}$ ) at the lowest velocities. The narrow features integrated over a relatively wide velocity range caused the integrated maps of the faint $\mathrm{c}-\mathrm{C}_{3} \mathrm{H}_{2}$ and $\mathrm{HC}_{3} \mathrm{~N}$ to be mostly dominated by noise. Thus, we included channel maps of integrated emission over $30 \mathrm{~km} \mathrm{~s}^{-1}$ bins for each species in Figs. 12 to 17 (online) where the origin of the fainter emission is clearly identified.

In Fig. 4 we labeled a number of selected positions corresponding to the molecular cores identified in the integrated maps. We extracted the spectra from each of these positions and performed a multiple velocity component Gaussian fit. The resulting parameters from these fits are shown in Table 3 (online) for $\mathrm{CN}$ and ${ }^{13} \mathrm{CN}$ and in Table 4 (online) for the rest of species.

\section{Spatially resolved chemistry}

The differences in the distribution of the emission of each of the detected species are shown in the integrated maps in Fig. 4. Strong differences are also evident in velocity, as shown in the channel maps in Figs. 12-17 (online). Below we describe 


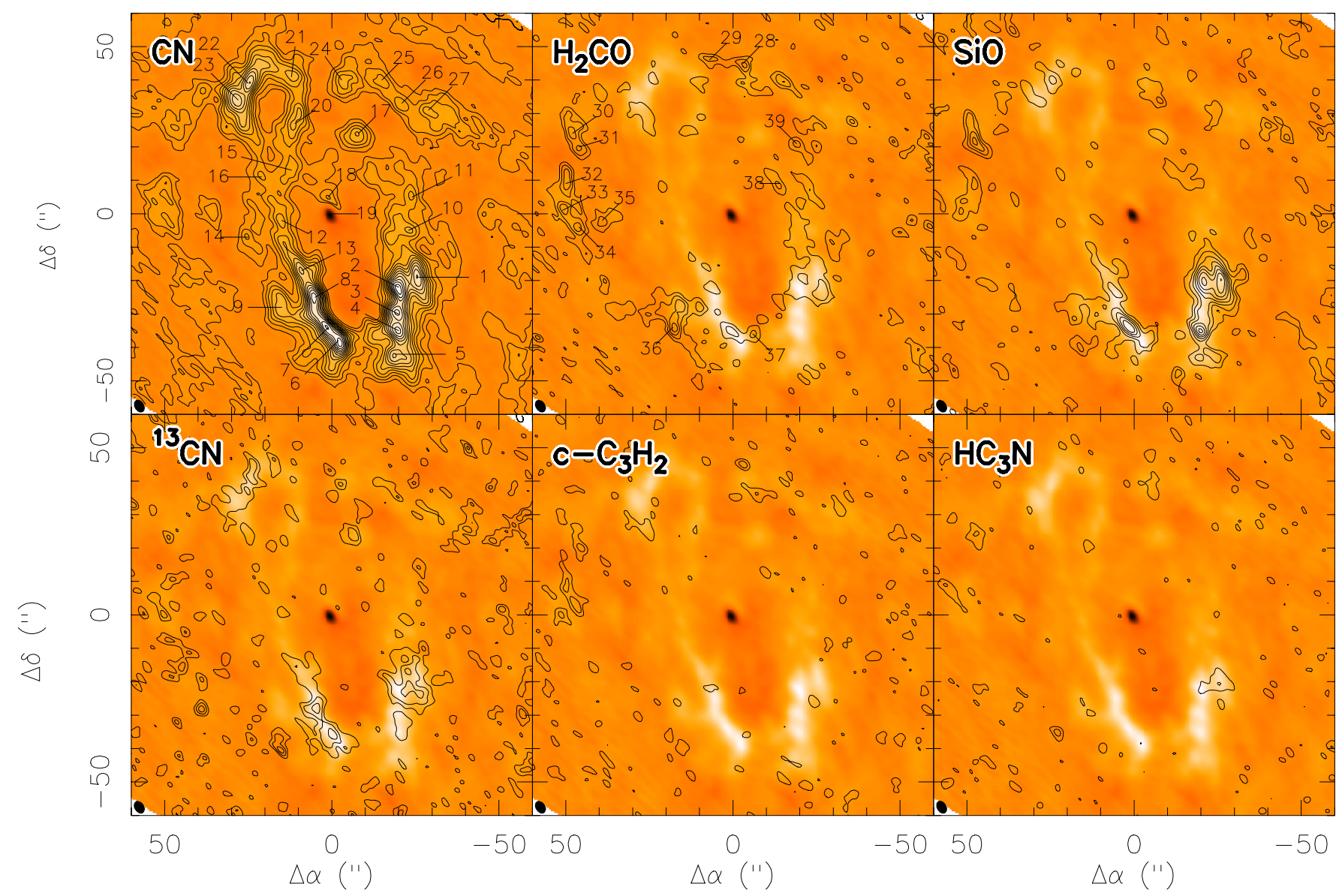

Fig. 4. Natural weighted integrated intensity maps of all detected species in this work. Beam size of $4.1^{\prime \prime} \times 2.9^{\prime \prime}$ is shown in the lower left corner of each box. Emission was integrated over the velocity range between -140 and $+140 \mathrm{~km} \mathrm{~s}^{-1}$ for $\mathrm{SiO}, \mathrm{C}_{3} \mathrm{H}_{2}$, and $\mathrm{HC}_{3} \mathrm{~N}$, while we used the velocity ranges of $[-190,+470]$ for $\mathrm{CN},[-80,+140]$ for $\mathrm{H}_{2} \mathrm{CO}$, and $[-190,+350]$ for ${ }^{13} \mathrm{CN}$ (see Sect. 3 for details). Background image corresponds to the integrated emission of CN. Contour levels are at $2 \sigma$ (with $\sigma=2.3 \mathrm{Jy} \mathrm{beam}^{-1} \mathrm{~km} \mathrm{~s}^{-1}$ ) steps for all maps but for CN, where the first contour is $3 \sigma$ and increases in $8 \sigma$ steps.

the main morphological structures observed for each individual species. The origin of each species is discussed in terms of chemical differentiation and excitation. To study the chemical differentiation in detail, we show line ratio maps in $30 \mathrm{~km} \mathrm{~s}^{-1}$ velocity bins in Figs. 18-22 (online).

The labeling of the different CND molecular structures, shown in Fig. 2, are adopted from Christopher et al. (2005) and Montero-Castaño et al. (2009).

\subsection{CN: overall molecular emission}

Out of the six molecular species observed, $\mathrm{CN}$ is the only one tracing all previously identified CND features. Though differences are observed, the integrated emission of $\mathrm{CN}$ appears to be mostly coexisting and tracing the same molecular material as that observed in HCN and CS (Christopher et al. 2005; MonteroCastaño et al. 2009). In Fig. 5 we present a comparison between the HCN 4-3 integrated emission from Montero-Castaño et al. (2009) and that of CN 2-1. Most of the main formation paths of $\mathrm{CN}$ in gas phase involve $\mathrm{HCN}$, either by direct photodissociation of $\mathrm{HCN}$ into $\mathrm{CN}$, charge exchange with $\mathrm{CN}^{+}$, ion-neutral reactions with $\mathrm{HCN}^{+}$and $\mathrm{C}_{2} \mathrm{H}^{+}$or by dissociative recombination of $\mathrm{HCN}^{+}$(Woodall et al. 2007). These processes are expected to be efficient because of the enhancement of ion species by the radiation, which pervades the environment surrounding the central cluster. Moreover, as shown in Fig. 6, $\mathrm{CN}$ is the most UV resistant species among those presented in this paper, with photodissociation rates for moderate shielding similar to those of $\mathrm{HCN}$ and CS. Indeed, CS formation is also known to be favored in UV radiated regions via the enhancement of its main precursor $\mathrm{S}^{+}$(Goicoechea et al. 2006, and references therein), which may explain why the three species show a similar spatial distribution in this region.

We can, however, point out a number of significant differences between the $\mathrm{HCN}$ and CS emission and that of the $\mathrm{CN}$ maps. The emission in the southwest lobe and southern extensions agree well in extension and location of the emission peaks of $\mathrm{CN}$ and $\mathrm{HCN}$. Even though $\mathrm{CN} 2-1$ and $\mathrm{HCN} 1-0$ transitions share similar excitation conditions, the southern extension observed in $\mathrm{CN}$ extends toward the north, connecting with the northern extension. This is observed in the 4-3 transition of HCN as well as in CS 7-6 (Montero-Castaño et al. 2009), but not in HCN 1-0 (Christopher et al. 2005). Therefore, this is not an effect of self-absorption of the HCN 1-0 in this extension toward the north but rather filtering of the extended emission in the OVRO maps by Christopher et al. (2005). The northeast extension and lobe appear connected in the CN map, similar to that observed in HCN 1-0. This connection is not observed in the 4-3 emission, likely because of a higher excitation in the southern part of the northeast extension as shown by the HCN 4-3/1-0 ratio in Fig. 8 from Montero-Castaño et al. (2009). The CN emission in the northeast arm is significantly 


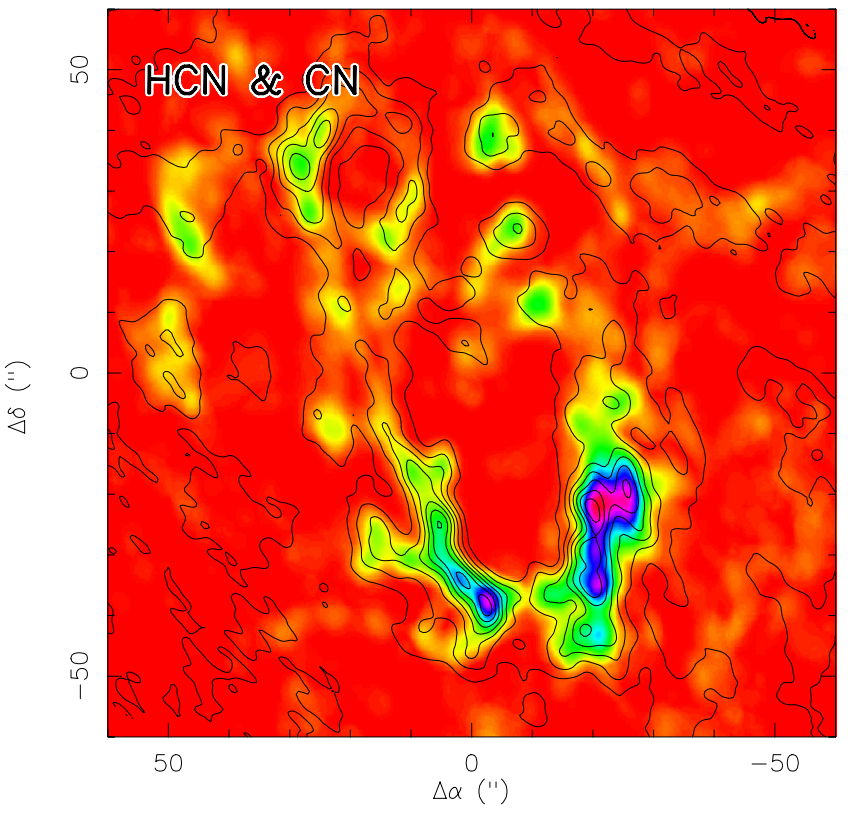

Fig. 5. Comparison between the HCN 4-3 integrated emission (color; Montero-Castaño et al. 2009) and the $\mathrm{CN}$ integrated emission (contours). Contour levels start at $3 \sigma$ and increases in $10 \sigma$ steps.

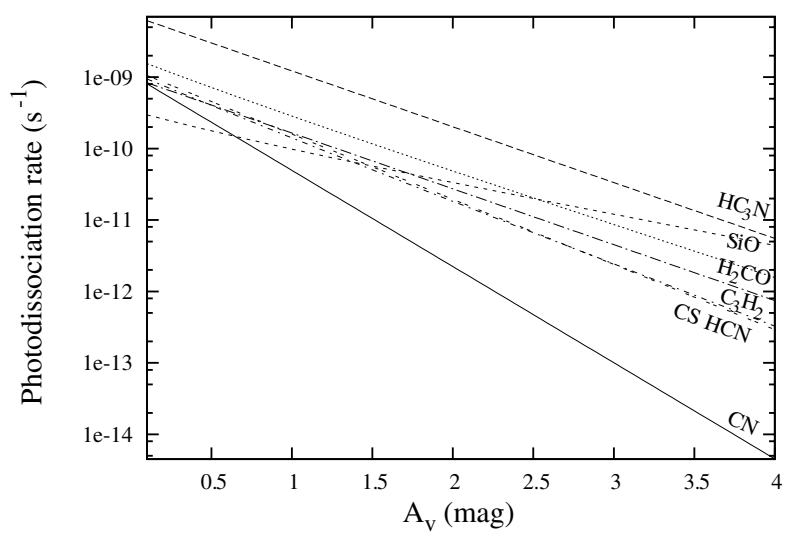

Fig. 6. Photodissociation rate as a function of the visual extinction for each of the species discussed. Rates are extracted from UMIST database (Woodall et al. 2007) and assume the standard ISM UV field (Draine 1978).

less prominent than that observed in $\mathrm{HCN}$, which might indicate a real abundance deficiency in this elongated feature. As discussed below (Sect. 4.2), this structure is prominent in $\mathrm{H}_{2} \mathrm{CO}$ and matches the emission structure traced by $\mathrm{HCN}$.

Based on the HCN 4-3 data of Montero-Castaño et al. (2009), at a similar resolution as the $\mathrm{CN} 2-1$ data, we derived some rough estimates of the $\mathrm{HCN} / \mathrm{CN}$ ratio of $\sim 3-2$ in the southern CND down to a slightly lower ratio of $\sim 2-1$ in the northeast lobe. The ratios were estimated by comparing the integrated intensity maps and confirmed by the comparison of the peak intensities of the spectra in selected positions shown in Fig. 4 from Montero-Castaño et al. (2009). These differences could be explained by the different excitation conditions along the CND region (Fig. 8 in Montero-Castaño et al. 2009).

\section{2. $\mathrm{H}_{2} \mathrm{CO}$ : expanding shell}

Figure 4 shows how the integrated intensity of $\mathrm{H}_{2} \mathrm{CO}$ differs drastically from that of $\mathrm{CN}$. Even though $\mathrm{H}_{2} \mathrm{CO}$ emission is detected toward the positions of peak $\mathrm{CN}$ emission (see Table 4 online), most of the emission of this molecule is detected in the outer regions of the CND. The emission of $\mathrm{H}_{2} \mathrm{CO}$ appears to trace a shell-like structure roughly centered at an offset position of $\sim\left(+12^{\prime \prime},+10^{\prime \prime}\right)$ from Sgr $A^{*}$ with a radius of $\sim 37^{\prime \prime}$. These differences are particularly evident in the channel maps in Fig. 13 (online). For the velocities below $0 \mathrm{~km} \mathrm{~s}^{-1}$, where significant high-velocity emission from $\mathrm{HC}_{3} \mathrm{~N} 24-23$ is detected in the northeast arm, the $\mathrm{H}_{2} \mathrm{CO}$ emission follows the brightest regions observed in $\mathrm{CN}$. At these velocities, $\mathrm{CN} / \mathrm{H}_{2} \mathrm{CO}$ line ratios range from 3-4 in the southwest lobe and in its connection to the southern extension (position 37 in Fig. 4) up to $>30$ in the regions where $\mathrm{H}_{2} \mathrm{CO}$ is not detected. However, the distribution of the emission differs for positive velocities. Channel maps centered at 0 and $30 \mathrm{~km} \mathrm{~s}^{-1}$ show emission mostly toward the south, while channel maps centered at 30 and $60 \mathrm{~km} \mathrm{~s}^{-1}$ show strong emission in the easternmost region of the maps. In these velocity channels the $\mathrm{H}_{2} \mathrm{CO}$ emission is observed to be extended over regions where $\mathrm{CN}$ is not detected, where line ratio limits $\mathrm{CN} / \mathrm{H}_{2} \mathrm{CO}<0.2$ are found. This is particularly prominent for the emission in the 0 and $30 \mathrm{~km} \mathrm{~s}^{-1}$ centered channels, where strong $\mathrm{H}_{2} \mathrm{CO}$ emission is observed east of the southern extension.

The $\mathrm{H}_{2} \mathrm{CO}$ molecule is efficiently formed in dust grains (Watanabe \& Kouchi 2002; Watanabe et al. 2004; Cuppen et al. 2009) where photo-induced chemistry by UV radiation can lead to the production of $\mathrm{H}_{2} \mathrm{CO}$ from the $\mathrm{H}_{2} \mathrm{O}$ and $\mathrm{CO}$ in solid phase (Shalabiea \& Greenberg 1994). Thus, the abundance of $\mathrm{H}_{2} \mathrm{CO}$ in the gas-phase can be enhanced owing to grain erosion by shocks and/or thermal evaporation in the vicinity of hot young stars (Shalabiea \& Greenberg 1994), while the main destruction paths are via photodissociation and reactions with $\mathrm{C}^{+}$ (Federman \& Allen 1991; Turner 1993). Indeed, after $\mathrm{HC}_{3} \mathrm{~N}$, the $\mathrm{H}_{2} \mathrm{CO}$ molecule is the most sensitive to photodissociation in our sample, which explains why, apart from the shell-like structure, $\mathrm{H}_{2} \mathrm{CO}$ is only detected closer to $\mathrm{Sgr} \mathrm{A}^{*}$ toward the brightest $\mathrm{CN}$ regions. In these dense regions the molecular gas is more shielded from photodissociation.

The observed circular structure could be interpreted as the interaction of Sgr A East mostly with the $50 \mathrm{~km} \mathrm{~s}^{-1}$ and $20 \mathrm{~km} \mathrm{~s}^{-1}$ giant molecular clouds, east and south of the CND, respectively. The kinematics of $\mathrm{H}_{2} \mathrm{CO}$ also support this scenario as seen in the channel maps. Taking the Gaussian fits in Table 4 (online), the brightest observed $\mathrm{H}_{2} \mathrm{CO}$ molecular components have velocities of $30-60 \mathrm{~km} \mathrm{~s}^{-1}$ toward the northern half of the CND with its maximum toward the northeast, and velocities of $\sim 10 \mathrm{~km} \mathrm{~s}^{-1}$ toward the southern regions. These velocities are consistent with the emission of $\mathrm{H}_{2} \mathrm{CO}$ being associated with an expanding shell impacting the $50 \mathrm{~km} \mathrm{~s}^{-1} \mathrm{GMC}$ in the north and northeast of the CND, and the $20 \mathrm{~km} \mathrm{~s}^{-1}$ GMC in the southern region. In these regions, $\mathrm{H}_{2} \mathrm{CO}$ abundance would be enhanced in the gas-phase by the expansion of Sgr A East into the surrounding GMCs, where it will survive in moderately dense molecular clumps $\left(A_{\mathrm{v}}>2\right.$, Shalabiea \& Greenberg 1994, and Fig. 6), shielded from the pervading UV radiation from the central cluster. The compression by an expanding shell is supported by the enhanced emission of $\mathrm{HC}_{3} \mathrm{~N}$ and $\mathrm{SiO}$ in this structure (Sects. 4.3 and 4.6).

Chemical differentiation of the eastern region is also suggested by the lower ${ }^{12} \mathrm{C} /{ }^{13} \mathrm{C}$ ratio measured in this region as compared to the central CND structure (see Sect. 4.4).

\subsection{SiO: infalling shocked material}

With a critical density of $\sim 10^{8} \mathrm{~cm}^{-3}$, the 5-4 transition of $\mathrm{SiO}$ is tracing the densest molecular material in the CND. The 
single-dish maps of the $\mathrm{SiO} 2-1$ (Amo-Baladrón et al. 2011) show the $\mathrm{SiO}$ emission to be extended and tracing the whole CND structure. Similarly, the interferometric map of $\mathrm{SiO} 2-1$ by Sato \& Tsuboi (2009) clearly shows emission along the CND. However, our map of the 5-4 transition emission is observed to be more compact as expected because of the different excitation conditions between both transitions. Indeed, the one order of magnitude larger critical density of the 5-4 transition compared to that of the 2-1 makes it unlikely that the emission is spread over large scales (see Sect. 2), and therefore filtered out in our maps.

SiO 5-4 emission is mostly found toward the southern region of the CND, in the southwest and southern extension, and the northeast arm. We find $\mathrm{CN} / \mathrm{SiO}$ line ratios ranging between $1-5$ in the southern region and of $\sim 8-10$ in the northeast. Similar to $\mathrm{H}_{2} \mathrm{CO}$, lower limits of $>30$ are found in the regions with no $\mathrm{SiO}$ detection. $\mathrm{SiO}$ is also observed in the northeast arm and east of the southern extension, where prominent $\mathrm{H}_{2} \mathrm{CO}$ emission was found, with limits to the $\mathrm{CN} / \mathrm{SiO}<0.3$ in the regions with no CN detection.

The SiO molecule is not efficiently formed in the cold gas phase if $\mathrm{Si}$ is not released to gas phase. Therefore, its emission is commonly accepted to be a tracer of the presence of shocks that are responsible for $\mathrm{SiO}$ ejection from grain mantles (Ziurys et al. 1989; Martin-Pintado et al. 1992). Indeed, SiO is widely spread across the Galactic center region likely because of large-scale shocks that affect the giant molecular clouds (MartinPintado et al. 1997). Though $\mathrm{SiO}$ has been found to be correlated with the $6.4 \mathrm{keV}$ Fe line, which points to an X-ray induced enhancement of SiO (Martín-Pintado et al. 2000; Amo-Baladrón et al. 2009), the lack of prominent $6.4 \mathrm{keV}$ line emission in the CND region (Yusef-Zadeh et al. 2007) and the compact emission of $\mathrm{SiO}$ makes it unlikely that $\mathrm{X}$-ray is the main driver of the high $\mathrm{SiO}$ abundance. Though UV photodesorption has been proposed as a possible driver of the $\mathrm{SiO}$ enhancement (Schilke et al. 2001), one would expect its distribution to be more uniform within the central CND, similar to $\mathrm{CN}$ and $\mathrm{HCN}$, and not so prominent in the northeast arm. Moreover, although it was claimed that shock velocities $>40 \mathrm{~km} \mathrm{~s}^{-1}$ are needed to destroy the silicate grains (Seab \& Shull 1983), recent observations and modeling have shown low-velocity C-shocks $\left(v=5-10 \mathrm{~km} \mathrm{~s}^{-1}\right)$ to efficiently sputter the silicon from grain mantles (JiménezSerra et al. 2005, 2008). Consequently, the enhancement of SiO toward the southern region could be understood by shock destruction of dust grains in the cloud complexes (Amo-Baladrón et al. 2011). From this material, the 5-4 transition is tracing the densest molecular material affected by shocks. These shocks could originate in gas inflow into the CND.

Coil \& Ho (1999) found kinematic evidence of gas streamers from the southern $20 \mathrm{~km} \mathrm{~s}^{-1}$ molecular cloud into the CND. The main gas flow, known as the southern streamer, would be connecting to the southern extension and could currently be feeding this molecular structure. Moreover, Coil \& Ho (1999) propose the presence of two streamers emerging from the $20 \mathrm{~km} \mathrm{~s}^{-1}$ cloud. The second streamer would originate in the northwest of the $20 \mathrm{~km} \mathrm{~s}^{-1}$ cloud and would connect the CND toward the southwest lobe. The SiO 2-1 map by Sato \& Tsuboi (2009) shows emission from two filaments south of the CND, which supports the idea of the two streamers. The presence of these streamers connecting the $20 \mathrm{~km} \mathrm{~s}^{-1}$ cloud with the CND would explain the two separated molecular complexes observed in the southern $\mathrm{CND}$ and the enhancement of $\mathrm{SiO}$ emission through shocked material in the interaction between the streamers and the southern structures. This gas infall might be the cause for the

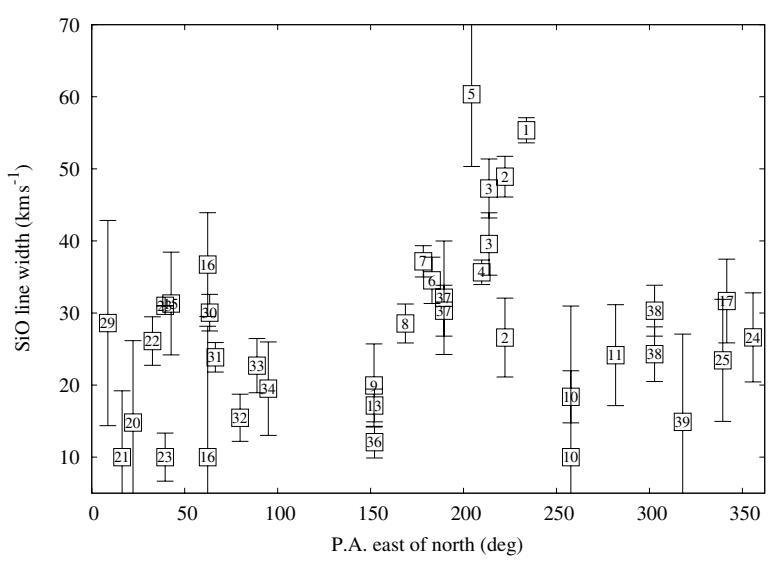

Fig. 7. $\mathrm{SiO}$ line width as a function of the position angle on the sky east of north as measured from Sgr A*. Numbers correspond to Fig. 4 labels. Position 14 and one of the velocity components in position 9 are not shown because of the large uncertainty in the width measurement. The positions with the larger widths at PA around $200^{\circ}$ correspond to the southern lobe and southern extension of the CND structure.

wider line widths observed toward the southern lobe. Figure 7 shows the measured line widths on each of the molecular components studied, where we observed the line widths to vary between 10 and $30 \mathrm{~km} \mathrm{~s}^{-1}$ in most positions except for those in the southern regions of the CND, where line widths systematically exceed $30 \mathrm{~km} \mathrm{~s}^{-1}$, up to values of $60 \mathrm{~km} \mathrm{~s}^{-1}$. This increase in the linewidth is consistent with the one observed in $\mathrm{NH}_{3}$ as the gas approaches the CND from the $20 \mathrm{~km} \mathrm{~s}^{-1}$ GMC (Coil \& Ho 1999). We note how the $\mathrm{SiO}$ emission, although it follows the kinematics of CN (see Sect. 6), is observed to be at the outer edge of the southwest lobe as seen in the -120 to $-90 \mathrm{~km} \mathrm{~s}^{-1}$ channel maps (Fig. 19 online), while the $\mathrm{SiO}$ enhanced gas is merged with the gas traced by $\mathrm{CN}$ in the -30 and $0 \mathrm{~km} \mathrm{~s}^{-1}$ channel maps. Similarly, the $\mathrm{SiO}$ emission in the southern extension is observed toward the southern edge of the structure. Toward the northeast lobe we also observe an enhancement of the $\mathrm{SiO}$ emission right at the center of this structure, which could be linked to the $50 \mathrm{~km} \mathrm{~s}^{-1}$ streamer from the nearby GMC (Jackson et al. 1993). Though $\mathrm{SiO}$ enhancement is observed at the positions where the inflowing streamers are claimed to connect to the CND, to fully confirm the inflowing origin of the $\mathrm{SiO}$ emission, we need largescale sensitive mapping, such as the one carried out towards the southern part of the CND by Sato \& Tsuboi (2009).

Additionally, we observe $\mathrm{SiO}$ emission in the northeast arm and east of the southern extension closely following the emission observed in $\mathrm{H}_{2} \mathrm{CO}$ (see Sect. 4.2). Silicon sputtering is expected at these positions in the scenario of Sgr A East expanding against the $50 \mathrm{~km} \mathrm{~s}^{-1}$ and $20 \mathrm{~km} \mathrm{~s}^{-1}$ GMCs.

We emphasize that the line widths of $\mathrm{SiO}$ at all measured positions do not show significant differences, within the measurement errors, with the line widths of the other species. Moreover, we did not find traces of shock-induced differences in the line profiles, such as line wings. Even if the velocity resolution of our maps is not sufficient to detect asymmetries caused by lowvelocity C-shocks, we can discard high-velocity shocks as the main origin of the $\mathrm{SiO}$ abundance. Thus, $\mathrm{SiO}$ is likely enhanced by cloud-cloud collisions as the material approaches the CND.

\section{4. ${ }^{13} \mathrm{CN}$ : carbon ${ }^{12} \mathrm{C} /{ }^{13} \mathrm{C}$ isotopic ratio}

The simultaneous observation of ${ }^{12} \mathrm{CN}$ and ${ }^{13} \mathrm{CN}$ provides a direct measurement of the ${ }^{12} \mathrm{C} /{ }^{13} \mathrm{C}$ isotopic ratio in the inner region of the Galaxy. Additionally, the fit of the hyperfine 
molecular components to the observed profiles allows us to estimate the opacity of the $\mathrm{CN}$ emission along the CND. Table 3 (online) shows the results of the fit to the $\mathrm{CN}$ and ${ }^{13} \mathrm{CN}$ observed profiles in a number of selected positions labeled in Fig. 4. The opacity of the brightest component of the $\mathrm{CN}$ hyperfine group, derived from the observed line profiles, is also tabulated in Table 3 (online). We find that $\mathrm{CN}$ is optically thick $(\tau \gtrsim 1)$ throughout most of the CND structure. We calculated the integrated intensity ratio $\mathrm{CN} /{ }^{13} \mathrm{CN}$ in all positions where ${ }^{13} \mathrm{CN}$ was detected.

From our estimate of the opacity of $\mathrm{CN}$, we calculated the opacity-corrected $\mathrm{CN} /{ }^{13} \mathrm{CN}$ as a proxy to the ${ }^{12} \mathrm{C} /{ }^{13} \mathrm{C}$ isotopic ratio. As shown by comparing the last two columns in Table 3 (online) and given that only the brightest hyperfine transitions within the observed $\mathrm{CN}$ spectral features are severely affected by opacity, the integrated intensity ratio $\mathrm{CN} /{ }^{13} \mathrm{CN}$ appears to provide a reasonably good estimate of the carbon isotopic ratio. We measured ${ }^{12} \mathrm{C} /{ }^{13} \mathrm{C}$ ratios in the wide range of $15-45$. This result agrees with the values reported toward the Sgr A region (Audouze et al. 1975; Wannier 1980) and is consistent with the scenario of ${ }^{13} \mathrm{C}$ enrichment toward the Galactic center region (Wilson \& Rood 1994; Wilson 1999).

Only a few positions are observed to have significantly low isotopic ratios $<10$. However, these positions are either particular velocity components that may not be related to the CND structure, or the eastern-most molecular component in our maps where the $\mathrm{H}_{2} \mathrm{CO}$ is most prominent. This difference in the isotopic ratio seems to support the $\mathrm{H}_{2} \mathrm{CO}$ molecular complex to be not only morphologically (Sect. 4.2), but chemically detached from the central CND structure. However, there are too few positions with measurements of this ratio to be able to draw firm conclusions based on the different carbon isotopic ratios.

\section{5. $\mathrm{C}-\mathrm{C}_{3} \mathrm{H}_{2}$}

Two spectral features of the carbon chain $\mathrm{c}-\mathrm{C}_{3} \mathrm{H}_{2}$ are detected. The one shown in Fig. 4, which is a combination of the $66_{1,6}-5_{0,5}$ and $6_{0,6}-5_{1,5}$ transitions, and a second feature centered at $217.940 \mathrm{GHz}$ corresponding to the $5_{1,4}-4_{2,3}$ transition. However, this feature is $\sim 2$ times fainter and is mostly undetected in our maps. This feature appears at the lowest velocities in the channel maps in Fig. 16 (online). Even at the positions where both features are detected, it is not possible to make a reliable estimate of the excitation temperature because of the limited dynamic range in the energies involved in these transitions.

The emission of $\mathrm{c}-\mathrm{C}_{3} \mathrm{H}_{2}$ is faint in the CND. It is barely visible in the integrated map (Fig. 4), but integrated channel maps (Fig. 16 online) show $\mathrm{c}-\mathrm{C}_{3} \mathrm{H}_{2}$ to follow $\mathrm{HC}_{3} \mathrm{~N}$. We note, however, that we found some selected positions where $\mathrm{c}-\mathrm{C}_{3} \mathrm{H}_{2}$ is detected, but no trace of $\mathrm{HC}_{3} \mathrm{~N}$ (see Table 4 online).

It has been claimed that $\mathrm{c}-\mathrm{C}_{3} \mathrm{H}_{2}$ is mainly formed in gas

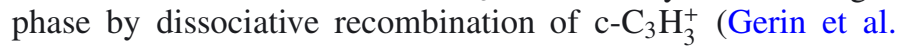
2011) and that it is much less sensitive to photodissociation than $\mathrm{HC}_{3} \mathrm{~N}$. This molecule has been observed to be very abundant in diffuse clouds (Lucas \& Liszt 2000) and in photodissociation regions (PDRs, Teyssier et al. 2004). Moreover, in their study of different PDRs, Teyssier et al. (2004) found the emission $\mathrm{c}-\mathrm{C}_{3} \mathrm{H}_{2}$ and other small hydrocarbons up to the edge of the PDR. On the chemical basis we would then expect the emission of $\mathrm{c}-\mathrm{C}_{3} \mathrm{H}_{2}$ to be more prominent over the entire CND region. In the Horsehead nebula, a column density ratio of c$\mathrm{C}_{3} \mathrm{H}_{2} / \mathrm{HC}_{3} \mathrm{~N} \sim 25 \pm 12$ is found in the position where both species are detected. Across the CND, we find integrated line ratios of c- $\mathrm{C}_{3} \mathrm{H}_{2} / \mathrm{HC}_{3} \mathrm{~N}=0.4-2.4$. Owing to the considerable upper level energy difference between the transitions observed for $\mathrm{c}-\mathrm{C}_{3} \mathrm{H}_{2}$ and $\mathrm{HC}_{3} \mathrm{~N}$, the column density ratio strongly depends on the excitation temperature. We assumed a range of likely temperatures of $20-50 \mathrm{~K}$, corresponding to the dust temperature components fitted by Etxaluze et al. (2011). We found column density ratios of $0.8-5 \times 10^{-2}$ and $0.3-1.6$ for a temperature of 20 and $50 \mathrm{~K}$, respectively. Thus, independent of the temperature assumption, abundance ratios in the CND differ significantly from those of PDR such as the Horsehead nebula. However, it is unclear whether this is caused by an underabundance of c$\mathrm{C}_{3} \mathrm{H}_{2}$ or by the enhanced emission of $\mathrm{HC}_{3} \mathrm{~N}$ in the CND region. Observations of the light carbon chain $\mathrm{CCH}$, intimately related to $\mathrm{c}-\mathrm{C}_{3} \mathrm{H}_{2}$ (Teyssier et al. 2004; Gerin et al. 2011), might be a better candidate to study the distribution of the carbon chains in the vicinity of Sgr A*.

\section{6. $\mathrm{HC}_{3} \mathrm{~N}$ : the warm dense molecular component}

Similar to $\mathrm{SiO} 5-4$, the $\mathrm{HC}_{3} \mathrm{~N} 25-24$ transition is likely tracing the densest molecular component with a critical density of $\sim 2 \times 10^{6} \mathrm{~cm}^{-3}$ (Wernli et al. 2007). Indeed, the emission of these two species appears to be coexisting and reaching its maximum emission toward the southern region of the CND (in the southwest lobe and the southern extension) and the northeast arm. Both species show the highest enhancement relative to $\mathrm{CN}$ in the northeast arm. We observed $\mathrm{CN} / \mathrm{HC}_{3} \mathrm{~N}$ ratios of $\sim 5-6$ in the southern structures down to ratios of $\sim 2$ in the northeast arm.

However, the formation and destruction of $\mathrm{HC}_{3} \mathrm{~N}$ differs from that of $\mathrm{SiO} . \mathrm{HC}_{3} \mathrm{~N}$ is efficiently formed in the gas phase by neutral-neutral reactions involving $\mathrm{CN}$ and $\mathrm{C}_{2} \mathrm{H}_{2}$, which can be abundant in an UV irradiated environment (Meier \& Turner 2005, and references therein). Additionally, $\mathrm{HC}_{3} \mathrm{~N}$ is the species with the highest photodissociation rate (Fig. 6) and is efficiently destroyed with reactions with $\mathrm{C}^{+}$. Therefore, in the heavily irradiated $\mathrm{CND}$ region, the formation reaction of $\mathrm{HC}_{3} \mathrm{~N}$ will overcome the photodestruction rate only within moderately shielded clouds $\left(A_{v}>1-2\right.$ Woodall et al. 2007).

Consequently, the high- $J$ transition of $\mathrm{HC}_{3} \mathrm{~N}$ with an upper level energy of $\sim 150 \mathrm{~K}$ is tracing the warmest dense molecular clumps, shielded from the photodissociation radiation from Sgr $\mathrm{A}^{*}$. In the southwest lobe and extension the $\mathrm{HC}_{3} \mathrm{~N}$ is observed in the outer edge of the $\mathrm{CN}$ emitting region, where the $\mathrm{UV}$ radiation is attenuated. $\mathrm{HC}_{3} \mathrm{~N}$ shows its brightest emission in the southern part of the northeast arm, where it shows the lowest ratio $\mathrm{SiO} / \mathrm{HC}_{3} \mathrm{~N}<1$. However, though $\mathrm{SiO}$ also traces the dense gas material, the formation of $\mathrm{HC}_{3} \mathrm{~N}$ via $\mathrm{CN}$ explains that no $\mathrm{HC}_{3} \mathrm{~N}$ emission is observed in regions where $\mathrm{CN}$ is absent, as seen in the $\mathrm{SiO}$ maps.

\section{The spectra toward Sgr $A^{*}$}

The low- $J$ transitions of HCN show absorption spectral features toward the position of Sgr A* (Guesten et al. 1987; Wright et al. 2001; Christopher et al. 2005). In Fig. 8 we show the CN absorption spectra toward the Sgr A* position.

We fitted a 3-component hyperfine group profile to the observed spectrum. In Fig. 8 we indicate the relative position and intensities of the $\mathrm{CN}$ hyperfine components and the fit results. The two narrow features at $-157 \mathrm{~km} \mathrm{~s}^{-1}$ and $-23 \mathrm{~km} \mathrm{~s}^{-1}$, with widths of 16 and $20 \mathrm{~km} \mathrm{~s}^{-1}$, respectively, have been previously identified as absorptions from galactic spiral arms along the line of sight at $-135,-50$ and $-30 \mathrm{~km} \mathrm{~s}^{-1}$ (see Wright et al. 2001). Our $-23 \mathrm{~km} \mathrm{~s}^{-1}$ feature is likely a composite of the extended local gas at $0 \mathrm{~km} \mathrm{~s}^{-1}$ (Guesten et al. 1987) and the -50 


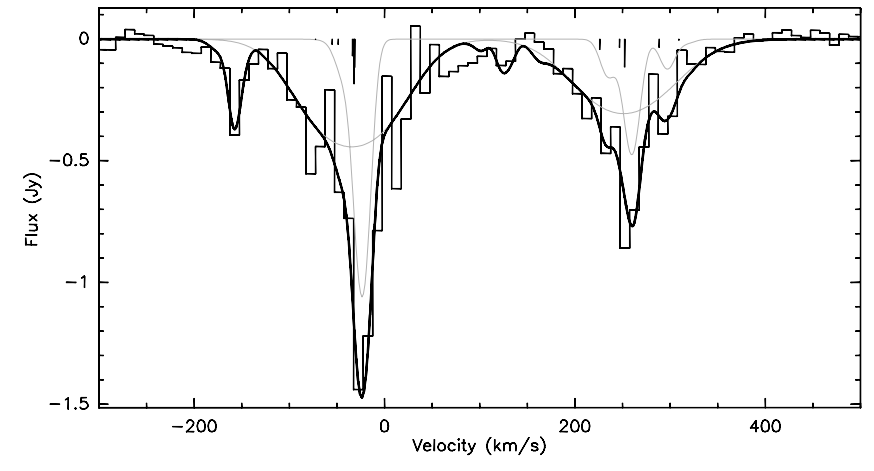

Fig. 8. Continuum-subtracted CN $2-1$ spectra extracted from a 4 " box around the Sgr A* position. The thick black line represents the fit performed to the line profile where three velocity components have been considered. Two narrow $\left(\sim 20 \mathrm{~km} \mathrm{~s}^{-1}\right)$ absorption features are fitted at -157 and $-23 \mathrm{~km} \mathrm{~s}^{-1}$ and a $\sim 100 \mathrm{~km} \mathrm{~s}^{-1}$ wide feature at $-32 \mathrm{~km} \mathrm{~s}^{-1}$. The thin gray line shows the individual contribution of the narrow $-23 \mathrm{~km} \mathrm{~s}^{-1}$ component and the wide component at $-32 \mathrm{~km} \mathrm{~s}^{-1}$. Vertical lines show the position and relative intensities of each individual CN hyperfine structure transition. The intensities and positions of these lines correspond to the fit results to the wider component.

and $-30 \mathrm{~km} \mathrm{~s}^{-1}$ galactic arms. The opacities derived from the fit for the main hyperfine transition are $\sim 0.1$ and 0.9 for the $-157 \mathrm{~km} \mathrm{~s}^{-1}$ and $-23 \mathrm{~km} \mathrm{~s}^{-1}$ components, respectively. Both narrow features are relatively optically thin.

On top of these narrow features we fitted a $94 \mathrm{~km} \mathrm{~s}^{-1}$ wide component centered at $-32 \mathrm{~km} \mathrm{~s}^{-1}$. Different from the narrow features, the opacity of the main hyperfine transition is $\tau_{\mathrm{CN}} \sim 3$. This is likely the material closer to Sgr A*, observed in absorption in the line of sight to the non-thermal central source emission from the low- $J$ of the transition. Down to zero level the absorption extends from $\sim-150 \mathrm{~km} \mathrm{~s}^{-1}$ to $\sim 100 \mathrm{~km} \mathrm{~s}^{-1}$, wider than the absorption observed from $\mathrm{HCN}$ or $\mathrm{HCO}^{+}$(Christopher et al. 2005). Moreover, the residual absorption in the velocity range around -200 and $100 \mathrm{~km} \mathrm{~s}^{-1}$ might suggest an even wider $\left(>100 \mathrm{~km} \mathrm{~s}^{-1}\right)$ absorption component.

The absorption spectrum toward the central compact sources is particularly interesting for studying the chemistry of the molecular gas in its line of sight. However, regarding the other molecular species presented in this paper, none is detected either in absorption or emission toward the Sgr A* position. This absence reflects abundance deficiencies of these species toward the galactic arms and also in the inner molecular gas around Sgr $\mathrm{A}^{*}$. The intense UV radiation from the central cluster is likely responsible for the photodissociation of most molecular species in the very central region.

\section{CND clump kinematics}

In this section we aim to analyze the kinematics of all molecular emission peaks selected and labeled in Fig. 4 including all velocity components fitted to each individual $\mathrm{CN}$ spectrum as compiled in Table 3 (online). We selected CN for this study because it is the only species that shows emission in all selected positions. A total of 66 velocity components were identified and fitted towards the 38 selected molecular clumps (where we do not include the central Sgr A* position).

Figure 9 shows the measured velocities as a function of the position angle (PA) east of north as measured from the position of Sgr A*. Similar plots have been presented by Guesten et al. (1987) and Jackson et al. (1993), who plotted the velocity centroids along the CND. However, we here include the individual velocity components at each selected position so that we can separate the different molecular clumps in velocity space in the line of sight. At each position we find molecular velocity components related to the different CND rotating structures as well as kinematically detached from it. Only $29 \%$ of all velocity components are associated with rotating structures around Sgr A* (see Sect. 6.1), while the remaining $\sim 70 \%$ belong to the overlapping GMCs or likely foreground material along the line of sight. However, these clumps in rotating structures represent $67 \%$ in mass of molecular gas. This estimate, based on the percentage of $\mathrm{CN}$ integrated intensity in the clumps, turns into a $70 \%-75 \%$ when corrected by the opacity of CN, mostly affecting the clumps associated with the rotating structures (Sect. 4.4).

\subsection{CND molecular components}

The CND is formed by distinct rotating structures and not a single structure (Jackson et al. 1993; Wright et al. 2001). Our observations seem to prove the presence of three kinematically distinct structures in the CND. We assumed a simple rotating ring model, similar to that used by Guesten et al. (1987), to describe the rotation around $\mathrm{Sgr} \mathrm{A}^{*}$ of each of these structures. Even though these structures are not complete rings, it is a reasonable approximation to describe the rotation in small position angle (PA) ranges.

The southern molecular structure of the CND (filled circles in Fig. 9) cannot be described by a single ring model. This could be observed in the data from Guesten et al. (1987). However, it is possible to fit two different ring models with inclinations of $\sim 80^{\circ}$ (solid line) and $\sim 68^{\circ}$ (dashed line) for the southwest lobe and the southern extension, respectively. The peak rotation velocity $\left(v_{\text {rot }} / \sin (i)\right)$ for these models were 125 and $86 \mathrm{~km} \mathrm{~s}^{-1}$, respectively. We observe that the southwest lobe model might extend northward up to position 11 , but would not fit position 10 (see Fig. 4). On the other hand, the southern extension model might extend eastward to positions 13 and 12, and toward the west to position 37 , which is located in the connecting point between the southern extension and the southwest lobe structures. These results suggest that though close in projection both southern structures do not belong to the same rotating structure, and indeed might be fed by different streamers (see Sect. 4.3).

Similarly, we can fit a ring model to the northeast lobe components (filled squares) with an inclination of $\sim 65^{\circ}$ and a peak velocity of $125 \mathrm{~km} \mathrm{~s}^{-1}$. This structure might start in positions 28 and 29, where $\mathrm{H}_{2} \mathrm{CO}$ shows a peak of emission, and would continue southward through positions 21 to 23 . The northeast extension (position 20, also in filled squares) is not sampled with sufficient points in our study, but it is clearly detached from the kinematics of the northeast lobe.

\subsection{Other molecular components}

The rest of the molecular components sampled do not seem to be associated with any evident rotating structure but rather with larger-scale structures placed on top of the CND.

We found a number of component at velocities of $\sim 50 \mathrm{~km} \mathrm{~s}^{-1}$ (open triangles). These positions are located mostly toward the north-east of the maps and are therefore likely related to gas condensations in the $50 \mathrm{~km} \mathrm{~s}^{-1}$ giant molecular cloud (see $\mathrm{SiO}$ and CS single-dish maps by Amo-Baladrón et al. 2011). Mostly toward the north-western parts of the CND we find a molecular component at $\sim 70 \mathrm{~km} \mathrm{~s}^{-1}$. As observed in the CS maps by 
S. Martín et al.: Surviving the hole. I.

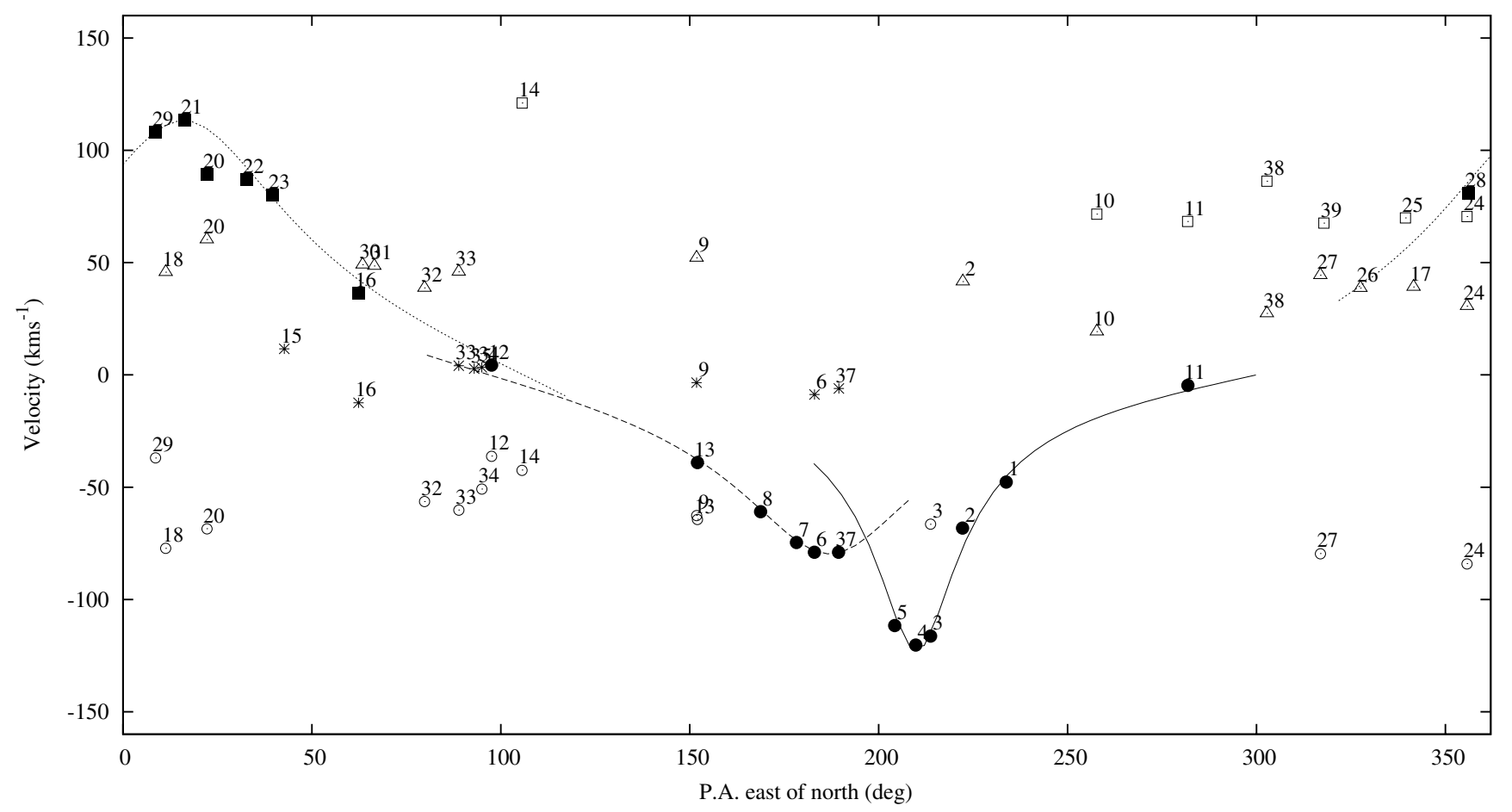

Fig. 9. Velocities of each of the CN molecular components fitted at each of the core positions labeled in Fig. 4 as a function of the position angle on the sky east of north as measured from Sgr $\mathrm{A}^{*}$. Each marker is labeled with the corresponding position where the velocity was measured. The different symbols represent different groups of cores/components: southern CND (filled circles), northern CND (filled squares), $70 \mathrm{~km} \mathrm{~s}^{-1}$ sources (open squares), $50 \mathrm{~km} \mathrm{~s}^{-1}$ sources (open triangles), $0 \mathrm{~km} \mathrm{~s}^{-1}$ sources (stars), and negative velocities sources (open circles). The overlaid lines represent rotating ring models fitted to the core velocities in the southwest lobe (solid line), southern extension (dashed line), and northeast lobe (dotted line). See Sect. 6 for details on the sources and models represented.

Amo-Baladrón et al. (2011), this could be an extension of the $50 \mathrm{~km} \mathrm{~s}^{-1}$ giant molecular cloud. At velocities of $\sim 0 \mathrm{~km} \mathrm{~s}^{-1}$ we find a number of components (stars in Fig. 9). These positions are mostly located toward the south-east of our maps and could be associated with the $20 \mathrm{~km} \mathrm{~s}^{-1} \mathrm{GMC}$ and the molecular bridge connecting to the $50 \mathrm{~km} \mathrm{~s}^{-1}$ GMC. Finally, we observed molecular gas at negative velocities (open circles). Mostly located toward the north-east of the CND, they are likely related to different molecular cloud complexes along the line of sight. However, there is no apparent connection with the CS maps by Amo-Baladrón et al. (2011).

Though the general trends described here for $\mathrm{CN}$ apply to all species, some significant differences are found in the velocity component fitted in the other detected species (see Table 4 online). In particular, the emitting region east of the southern extension (Sect. 4.2), where no $\mathrm{CN}$ is detected, shows emission peaks at the velocities of $\sim-9$ and $11 \mathrm{~km} \mathrm{~s}^{-1}$ (position 36 in Table 4 online) consistent with the molecular gas being associated with both foreground clouds and the $20 \mathrm{~km} \mathrm{~s}^{-1}$ GMC.

\section{CND structure and interaction with the mini-spiral}

The association of the gas in the CND with the ionized minispiral structure has long been discussed (Christopher et al. 2005, and references therein). The inner edge of the CND in the southwest lobe appears to be tightly outlined by the western ionized arc. This correlation has led to the idea that the western arc might be the ionized boundary of the CND where the strong pervasive UV field from the central cluster is responsible for the heating of the CND (Martín et al. 2008; Amo-Baladrón et al. 2011) and the mini-spiral structure (Latvakoski et al. 1999). This association was kinematically confirmed by Christopher et al. (2005). In Fig. 10 we compared our $\mathrm{CN}$ observations with the ionized gas traced by the H92 $\alpha$ emission from Roberts \& Goss (1993). We observe that not only is the emission tracing the inner edge of the southwest lobe, but the H92 $\alpha$ appears to trace the inner side facing Sgr A* of the CN emitting region. Similarly, the emission peaks of $\mathrm{SiO}$ in the southwest lobe are observed in the emission gaps of H92 $\alpha$ (lower panel in Fig. 10), as if the ionized gas were tracing the inner boundaries of the $\mathrm{SiO}$ emitting gas.

Christopher et al. (2005) noticed a possible connection of the northern arm of the mini-spiral and the northeastern extension of the CND. Our CN observations, in agreement with the HCN 4-3 maps by Montero-Castaño et al. (2009), show a more prominent emission in the northeast extension connecting to a faint but continuous eastern CND molecular emission. Again, the H92 $\alpha$ northern arm appears to be tracing the inner edge of the northeast extension of the CND, which might show a similar association as that claimed for the western arc.

We also observe an anti correlation between the ionized gas and the molecular material. The brightest northern arc outlines the inner edge of the faint molecular gas northeast extension, while the fainter western arc traces the edge of the bright molecular southwest lobe. This could be related to the age of each of the CND structures and the history of molecular fueling from the outer molecular clouds into the CND. In this scenario, the northeast extension would have been mostly ionized in the northern arm and little molecular gas would be left, while the southwest lobe, mostly molecular, would begin to become ionized as it approaches the central region.

The third mini-spiral arc, the eastern arm, has no obvious association with the molecular material. The low-resolution maps of HCN by Marshall et al. (1995) suggested this arc extends 


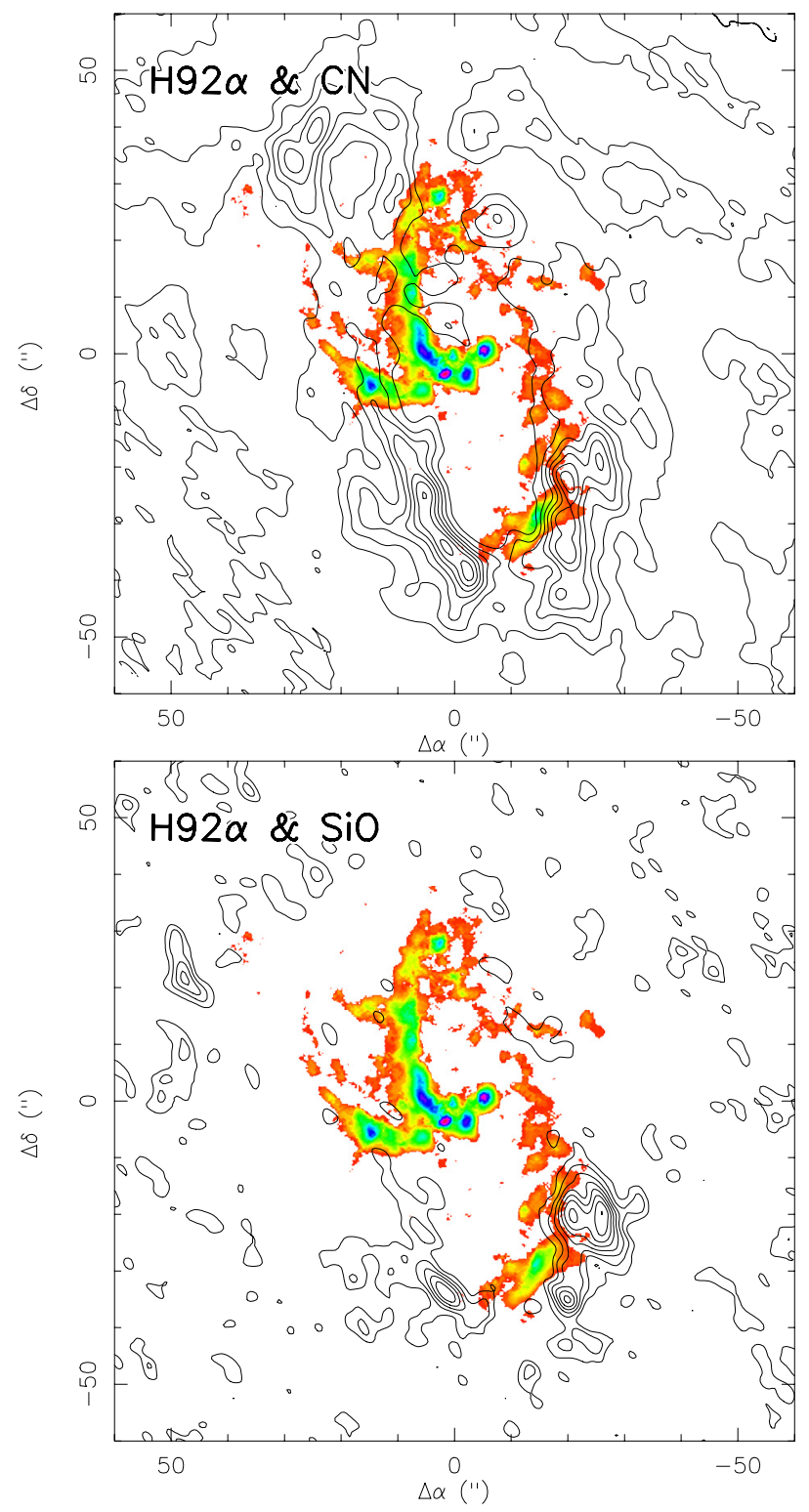

Fig. 10. (Upper) Comparison between the H92 $\alpha$ integrated emission (color; Roberts \& Goss 1993; Zhao et al. 2009) and the CN integrated emission (contours). CN contour levels are the same as in Fig. 5. (Lower) Comparison between the H92 $\alpha$ integrated emission (color; Roberts \& Goss 1993; Zhao et al. 2009) and the SiO integrated emission (contours). SiO contour levels are the same as in Fig. 4.

northward likely toward the north lobe. Indeed, at the position of $\sim\left(25^{\prime \prime}, 30^{\prime \prime}\right)$, the eastern arm traced by H92 $\alpha$ emission meets the northeast molecular lobe (upper panel in Fig. 10). Measured velocities from $\mathrm{H} 92 \alpha$ at this position (positions E1 and E2 in Zhao et al. 2009) match those in the northeast molecular lobe (positions 22 and 23 in Fig. 9). Similarly, the eastern ionized arm extends northward as seen in the $6 \mathrm{~cm}$ radio maps (Fig. 11, Yusef-Zadeh \& Morris 1987). Though this arm appears to be crossing the eastern extension of the CND, there is no evidence of perturbation of the molecular material, which indicates that both features are not in the same plane. Dust emission modeling (Latvakoski et al. 1999) and H92 $\alpha$ kinematics (Zhao et al. 2009) showed that the eastern arm must be significantly inclined with respect to the eastern $\mathrm{CND}$.

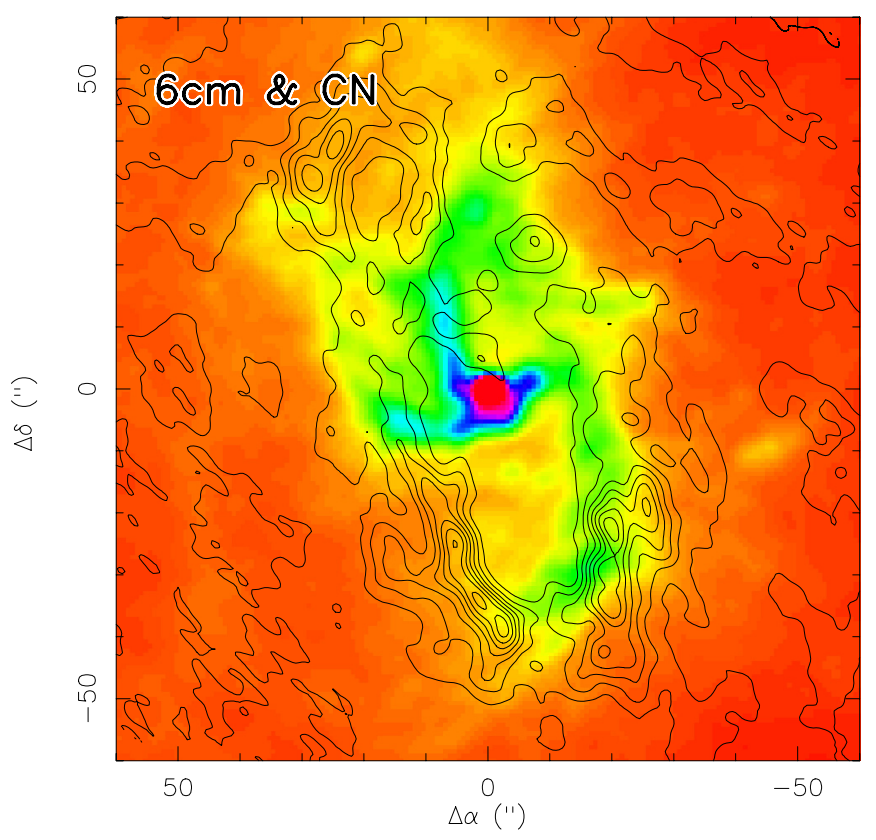

Fig. 11. Comparison between the $6 \mathrm{~cm}$ continuum emission (color; Yusef-Zadeh \& Morris 1987) and the CN integrated emission (contours). CN contour levels are the same as in Fig. 5. Similar to the comparison with $\mathrm{H} 92 \alpha$ in Fig. 10, the association of the northern arm and the western arc with the northern molecular extension and the southwest lobe can be appreciated. The $6 \mathrm{~cm}$ shows the clear extension of the eastern arm northward where it meets the bright molecular northeast lobe.

\section{Summary}

We presented high-resolution maps of the $\mathrm{CN},{ }^{13} \mathrm{CN}, \mathrm{H}_{2} \mathrm{CO}$, $\mathrm{SiO}, \mathrm{c}-\mathrm{C}_{3} \mathrm{H}_{2}$ and $\mathrm{HC}_{3} \mathrm{~N}$ emission toward the central $\sim 4 \mathrm{pc}$ around $\mathrm{Sgr} \mathrm{A}^{*}$. These observations provide a chemical picture of the molecular material in the surroundings of the central super-massive black hole and how the chemically resolved structures relate to the different morphological and kinematical structures. Each of the observed species appears to be tracing different molecular gas structures that are intimately linked to the different heating processes that affect the molecular gas in this region.

Among them, $\mathrm{CN}$ is the only species showing emission in all previously described molecular morphological features in the CND, and it shows a similar distribution to that from available $\mathrm{HCN}$ and CS emission maps (Christopher et al. 2005; MonteroCastaño et al. 2009). Similar to these two molecules, CN is extremely resistant against the high UV irradiation from the central cluster. Moreover, its chemistry is linked to that of HCN. These species are shown to be widespread at the closest distances from Sgr $A^{*}$. The emission from $\mathrm{H}_{2} \mathrm{CO}$ differs drastically from $\mathrm{CN}$ and it is mostly located in the outer regions of the CND, tracing a $\sim 37^{\prime \prime}$ shell-like structure centered $\sim 16^{\prime \prime}$ away from $\mathrm{Sgr} \mathrm{A}^{*}$ where $\mathrm{H}_{2} \mathrm{CO}$ is observed to be even brighter than $\mathrm{CN}$ (with $\mathrm{CN} / \mathrm{H}_{2} \mathrm{CO}$ line ratios below 0.2). Mostly formed in solid phase, $\mathrm{H}_{2} \mathrm{CO}$ emission in gas phase can be interpreted as the result of the expansion of Sgr A East against the $50 \mathrm{~km} \mathrm{~s}^{-1}$ and $20 \mathrm{~km} \mathrm{~s}^{-1}$ GMCs east and south of the CND, respectively. Moreover, the ${ }^{12} \mathrm{C} /{ }^{13} \mathrm{C}$ isotopic ratio, estimated from the ${ }^{12} \mathrm{CN} /{ }^{13} \mathrm{CN}$, supports the differentiation of the northeast arm, where the brightest $\mathrm{H}_{2} \mathrm{CO}$ emission is observed. While we estimated an opacity corrected carbon isotopic ratio in the range of 15-45 throughout the $\mathrm{CND}$, consistent with the idea of ${ }^{13} \mathrm{C}$ enrichment with decreasing 
galactocentric distance owing to nuclear processing (Wilson \& Rood 1994; Wilson 1999), we found the lowest measured ratios $(<10)$ toward the northeast arm. The opacity derived from the hyperfine fit to the $\mathrm{CN}$ spectral profiles shows that $\mathrm{CN}$ is optically thick throughout most of the CND. On the other hand, the densest $\left(n>10^{6} \mathrm{~cm}^{-3}\right)$ regions in the CND are located in the southern $\mathrm{CND}$ and the northeast arm as traced by $\mathrm{HC}_{3} \mathrm{~N}$ and $\mathrm{SiO}$, although their chemical formation paths are different. The southeast lobe and southern extension, where we find the highest $\mathrm{SiO}$ abundance and broader line widths, could be fueled and shocked by the molecular material from two streamers connecting the $20 \mathrm{~km} \mathrm{~s}^{-1} \mathrm{GMC}$, where $\mathrm{SiO}$ abundance would be enhanced through grain erosion by low-velocity shocks. Similarly, the bright emission in the northeast lobe could be the consequence of the inflowing material from the $50 \mathrm{~km} \mathrm{~s}^{-1}$ streamer connecting the GMC east of the CND. The similar line widths measured in all species allow us to discard high-velocity shocks as the origin of $\mathrm{SiO}$. The dense material in the northeast arm could be compressed because of the expansion of Sgr A East against the $50 \mathrm{~km} \mathrm{~s}^{-1} \mathrm{GMC}$, where the brightest $\mathrm{HC}_{3} \mathrm{~N}$ emission as well as significant $\mathrm{SiO}$ emission are detected. Even though $\mathrm{HC}_{3} \mathrm{~N}$ is efficiently formed through reactions involving $\mathrm{CN}$, the emission of $\mathrm{HC}_{3} \mathrm{~N}$ is deficient close to $\mathrm{Sgr} \mathrm{A}^{*}$ owing to the high photodissociation rate of this molecule in UV exposed material. The few regions in the inner CND where $\mathrm{HC}_{3} \mathrm{~N}$ emission is observed will be tracing the densest cores $\left(A_{\mathrm{v}}>1-2\right)$ for this molecule to survive. Finally, the carbon chain c- $\mathrm{C}_{3} \mathrm{H}_{2}$ is observed to follow the emission of $\mathrm{HC}_{3} \mathrm{~N}$, but with $\mathrm{c}-\mathrm{C}_{3} \mathrm{H}_{2} / \mathrm{HC}_{3} \mathrm{~N}$ at least an order of magnitude lower than in other Galactic PDRs. It is therefore unclear why the emission of $\mathrm{c}-\mathrm{C}_{3} \mathrm{H}_{2}$ is so faint in the CND.

In the direction of $\mathrm{Sgr} \mathrm{A}^{*}$ only $\mathrm{CN}$ is detected in absorption. It shows two previously identified narrow absorptions at -157 and $-23 \mathrm{~km} \mathrm{~s}^{-1}$ from galactic arms along the line of sight. A broad $94 \mathrm{~km} \mathrm{~s}^{-1}$ line at $-32 \mathrm{~km} \mathrm{~s}^{-1}$ is likely tracing the molecular gas closer to Sgr A* absorbing the non-thermal central emission. Moreover, we found hints of a possible wider $\left(>100 \mathrm{~km} \mathrm{~s}^{-1}\right) \mathrm{ab}-$ sorption, which might be tracing the closest molecular material to the central black hole. However, observations of higher energy transitions of UV-resistant species are required to detect this molecular gas component in emission as shown by the HCN 4-3 maps by Montero-Castaño et al. (2009).

We have studied the kinematics of all detected molecular clumps in the CND, where we considered kinematics of each individual velocity component at every selected position. Our study shows that most of these clumps are not associated with any rotating structure around $\mathrm{Sgr} \mathrm{A}^{*}$ but with larger-scale structures overlapping with the CND. However, even if only $29 \%$ of the molecular selected components appear clearly associated with rotating structures, they represent $70-75 \%$ of the mass of the molecular gas. Only three molecular structures show kinematic evidence of rotation around Sgr $\mathrm{A}^{*}$. These rotating filaments, namely the southwest lobe, southern extension, and northeast lobe, are rotating in different planes, similar to what is derived from the study of the ionized arcs (Zhao et al. 2009), and are associated with the ionized mini-spiral structure observed in radio continuum and hydrogen recombination lines.

Moreover, we have shown how the various chemically differentiated structures are directly related to the different kinematical structures. In this comparison we observe how $\mathrm{CN}$ is mostly concentrated in the dense molecular optically thick clumps rotating (and likely infalling) filaments around $\mathrm{Sgr} \mathrm{A}^{*}$, while the shell-like structure traced by $\mathrm{H}_{2} \mathrm{CO}$ is not orbiting around the central black hole, but shows velocities related to the overlapping GMCs.

We showed the potential of detailed high-resolution chemical observations to distinguish the complicated molecular structure surrounding $\mathrm{Sgr} \mathrm{A}^{*}$, and to establish the leading heating mechanisms that affect the different molecular structures in the region. Similar to the low-resolution work by Amo-Baladrón et al. (2011), chemical abundances from deep high-resolution imaging of various molecular species as a function of position and distance from Sgr A* could potentially be used to establish the three-dimensional distribution of the molecular components in the Galactic center region.

Acknowledgements. J.M.-P. and S.M. have been supported MICINN through grants ESP2007-65812-C02-C01 and AYA2010-21697-C05-01, and AstroMadrid (CAM S2009/ESP-1496). The authors would like to thank the referee, whose comments helped to significantly improve the paper.

\section{References}

Amo-Baladrón, M. A., Martín-Pintado, J., Morris, M. R., Muno, M. P., \& Rodríguez-Fernández, N. J. 2009, ApJ, 694, 943

Amo-Baladrón, M. A., Martín-Pintado, J., \& Martín, S. 2011, A\&A, 526, A54

Audouze, J., Lequeux, J., \& Vigroux, L. 1975, A\&A, 43, 71

Bélanger, G., Goldwurm, A., Renaud, M., et al. 2006, ApJ, 636, 275

Christopher, M. H., Scoville, N. Z., Stolovy, S. R., \& Yun, M. S. 2005, ApJ, 622, 346

Coil, A. L., \& Ho, P. T. P. 1999, ApJ, 513, 752

Cuppen, H. M., van Dishoeck, E. F., Herbst, E., \& Tielens, A. G. G. M. 2009, A\&A, 508, 275

Davidson, J. A., Werner, M. W., Wu, X., et al. 1992, ApJ, 387, 189

Draine, B. T. 1978, ApJS, 36, 595

Ekers, R. D., van Gorkom, J. H., Schwarz, U. J., \& Goss, W. M. 1983, A\&A, 122,143

Etxaluze, M., Smith, H. A., Tolls, V., Stark, A. A., \& González-Alfonso, E. 2011, AJ, 142, 134

Federman, S. R., \& Allen, M. 1991, ApJ, 375, 157

Gerin, M., Kaźmierczak, M., Jastrzebska, M., et al. 2011, A\&A, 525, A116

Ghez, A. M., Salim, S., Weinberg, N. N., et al. 2008, ApJ, 689, 1044

Goicoechea, J. R., Pety, J., Gerin, M., et al. 2006, A\&A, 456, 565

Groenewegen, M. A. T., Udalski, A., \& Bono, G. 2008, A\&A, 481, 441

Guesten, R., Genzel, R., Wright, M. C. H., et al. 1987, ApJ, 318, 124

Herrnstein, R. M., \& Ho, P. T. P. 2005, ApJ, 620, 287

Ho, P. T. P., Ho, L. C., Szczepanski, J. C., Jackson, J. M., \& Armstrong, J. T. 1991, Nature, 350, 309

Ho, P. T. P., Moran, J. M., \& Lo, K. Y. 2004, ApJ, 616, L1

Jackson, J. M., Geis, N., Genzel, R., et al. 1993, ApJ, 402, 173

Jiménez-Serra, I., Martín-Pintado, J., Rodríguez-Franco, A., \& Martín, S. 2005, ApJ, 627, L121

Jiménez-Serra, I., Caselli, P., Martín-Pintado, J., \& Hartquist, T. W. 2008, A\&A, 482,549

Lacy, J. H., Townes, C. H., Geballe, T. R., \& Hollenbach, D. J. 1980, ApJ, 241, 132

Latvakoski, H. M., Stacey, G. J., Gull, G. E., \& Hayward, T. L. 1999, ApJ, 511, 761

Lo, K. Y., \& Claussen, M. J. 1983, Nature, 306, 647

Lucas, R., \& Liszt, H. S. 2000, A\&A, 358, 1069

Marr, J. M., Wright, M. C. H., \& Backer, D. C. 1993, ApJ, 411, 667

Marshall, J., Lasenby, A. N., \& Harris, A. I. 1995, MNRAS, 277, 594

Martín, S., Requena-Torres, M. A., Martín-Pintado, J., \& Mauersberger, R. 2008, ApJ, 678, 245

Martin-Pintado, J., Bachiller, R., \& Fuente, A. 1992, A\&A, 254, 315

Martin-Pintado, J., de Vicente, P., Fuente, A., \& Planesas, P. 1997, ApJ, 482, L45

Martín-Pintado, J., de Vicente, P., Rodríguez-Fernández, N. J., Fuente, A., \& Planesas, P. 2000, A\&A, 356, L5

McGary, R. S., Coil, A. L., \& Ho, P. T. P. 2001, ApJ, 559, 326

Meier, D. S., \& Turner, J. L. 2005, ApJ, 618, 259

Montero-Castaño, M., Herrnstein, R. M., \& Ho, P. T. P. 2006, J. Phys. Conf. Ser., 54,29

Montero-Castaño, M., Herrnstein, R. M., \& Ho, P. T. P. 2009, ApJ, 695, 1477

Reid, M. J. 1993, ARA\&A, 31, 345

Roberts, D. A., \& Goss, W. M. 1993, ApJS, 86, 133 
A\&A 539, A29 (2012)

Sato, M. T., \& Tsuboi, M. 2009, in Approaching Micro-Arcsecond Resolution with VSOP-2: Astrophysics and Technologies, ed. Y. Hagiwara, E. Fomalont,

M. Tsuboi, \& M. Yasuhiro, ASP Conf. Ser., 402, 388

Sault, R. J., Teuben, P. J., \& Wright, M. C. H. 1995, in Astronomical Data Analysis Software and Systems IV, ed. R. A. Shaw, H. E. Payne, \& J. J. E. Hayes, ASP Conf. Ser., 77, 433

Schilke, P., Pineau des Forêts, G., Walmsley, C. M., \& Martín-Pintado, J. 2001, A\&A, 372, 291

Seab, C. G., \& Shull, J. M. 1983, ApJ, 275, 652

Shalabiea, O. M., \& Greenberg, J. M. 1994, A\&A, 290, 266

Teyssier, D., Fossé, D., Gerin, M., et al. 2004, A\&A, 417, 135

Turner, B. E. 1993, ApJ, 410, 140

Wannier, P. G. 1980, ARA\&A, 18, 399
Watanabe, N., \& Kouchi, A. 2002, ApJ, 571, L173

Watanabe, N., Nagaoka, A., Shiraki, T., \& Kouchi, A. 2004, ApJ, 616, 638

Wernli, M., Wiesenfeld, L., Faure, A., \& Valiron, P. 2007, A\&A, 464, 1147

Wilson, T. L. 1999, Rep. Prog. Phys., 62, 143

Wilson, T. L., \& Rood, R. 1994, ARA\&A, 32, 191

Woodall, J., Agúndez, M., Markwick-Kemper, A. J., \& Millar, T. J. 2007, A\&A, 466, 1197

Wright, M. C. H., Coil, A. L., McGary, R. S., Ho, P. T. P., \& Harris, A. I. 2001, ApJ, 551, 254

Yusef-Zadeh, F., \& Morris, M. 1987, ApJ, 320, 545

Yusef-Zadeh, F., Muno, M., Wardle, M., \& Lis, D. C. 2007, ApJ, 656, 847

Zhao, J., Morris, M. R., Goss, W. M., \& An, T. 2009, ApJ, 699, 186

Ziurys, L. M., Friberg, P., \& Irvine, W. M. 1989, ApJ, 343, 201 
S. Martín et al.: Surviving the hole. I.

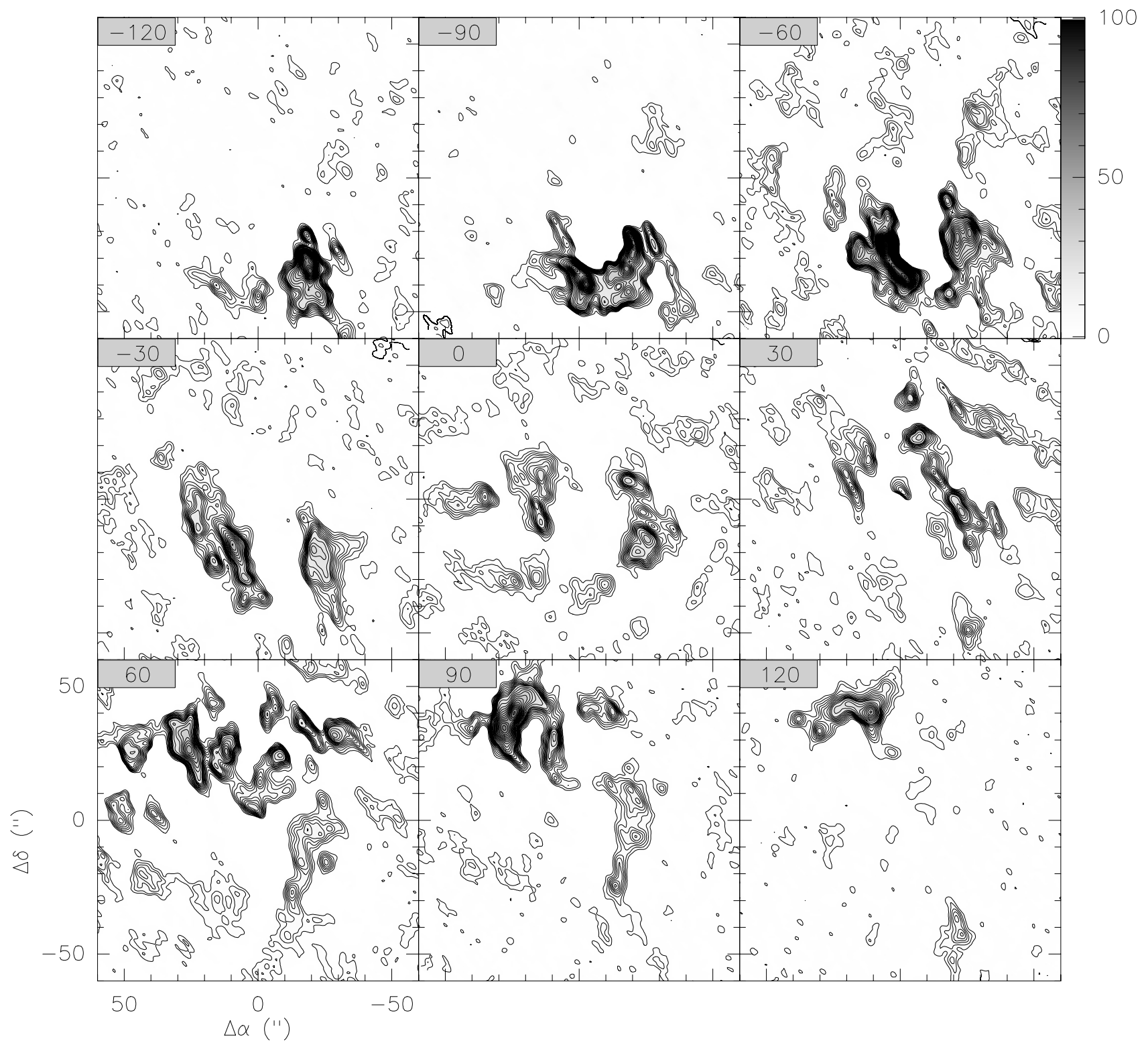

Fig. 12. Integrated intensity maps of $\mathrm{CN} 2-1$ in velocity bins of $30 \mathrm{~km} \mathrm{~s}^{-1}$. Central velocity of each velocity range is displayed in the upper left corner of each panel. First 10 contours are in steps of $3 \sigma$, with $\sigma=0.55 \mathrm{Jy}_{\text {beam }}^{-1} \mathrm{~km} \mathrm{~s}^{-1}$, and subsequently in steps of $6 \sigma$. 
A\&A 539, A29 (2012)

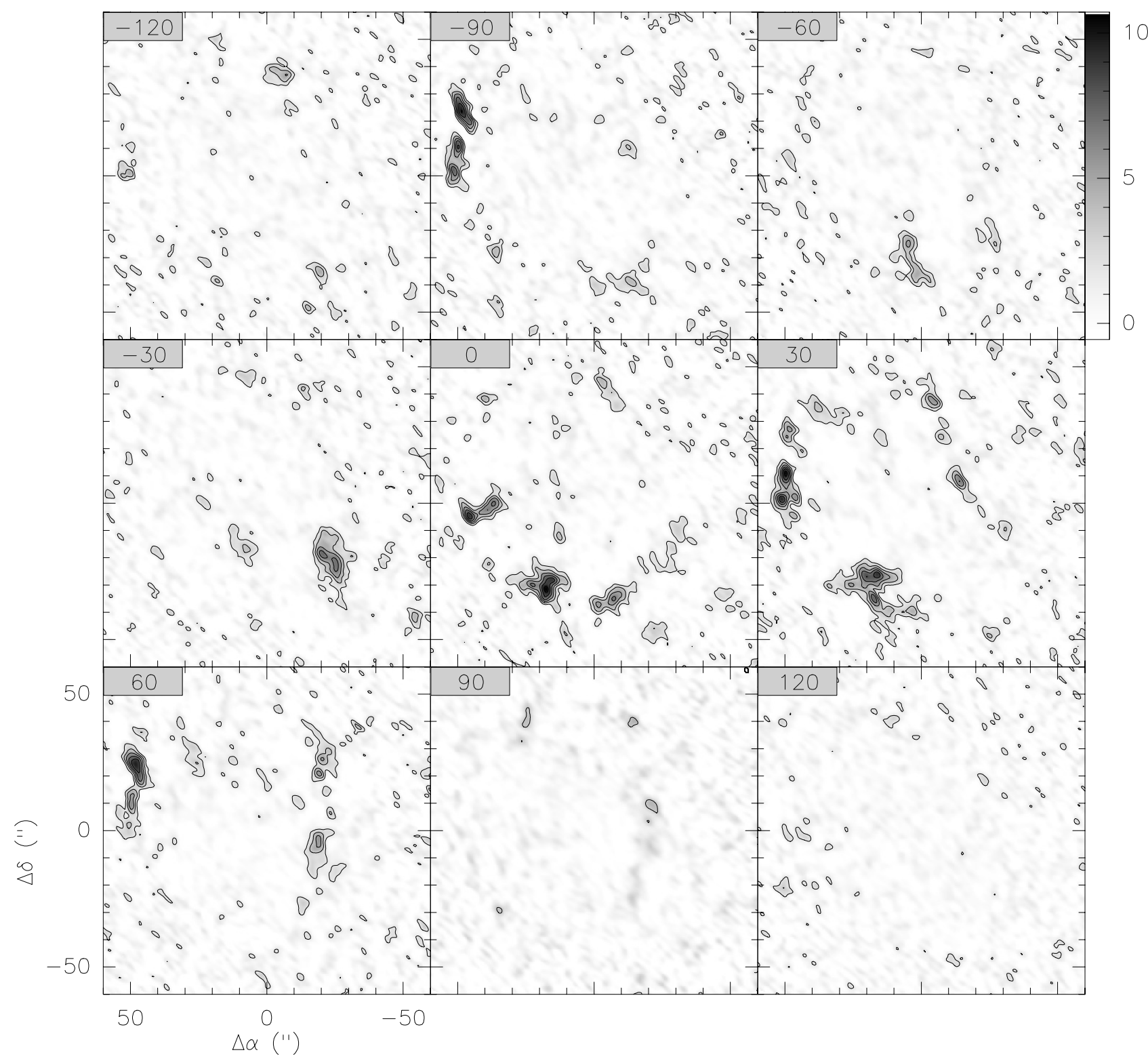

Fig. 13. Integrated intensity maps of $\mathrm{H}_{2} \mathrm{CO} 3_{0,3}-2_{0,2}$ in velocity bins of $30 \mathrm{~km} \mathrm{~s}^{-1}$. Labels and contour steps as in Fig. 12. Note that the observed emission toward the north and east in the -120 and $-90 \mathrm{~km} \mathrm{~s}^{-1}$ maps correspond to the high-velocity emission of $\mathrm{HC}_{3} \mathrm{~N} 24-23(218.324 \mathrm{GHz})$ detected at the edge of the observed band (Sect. 3). 
S. Martín et al.: Surviving the hole. I.

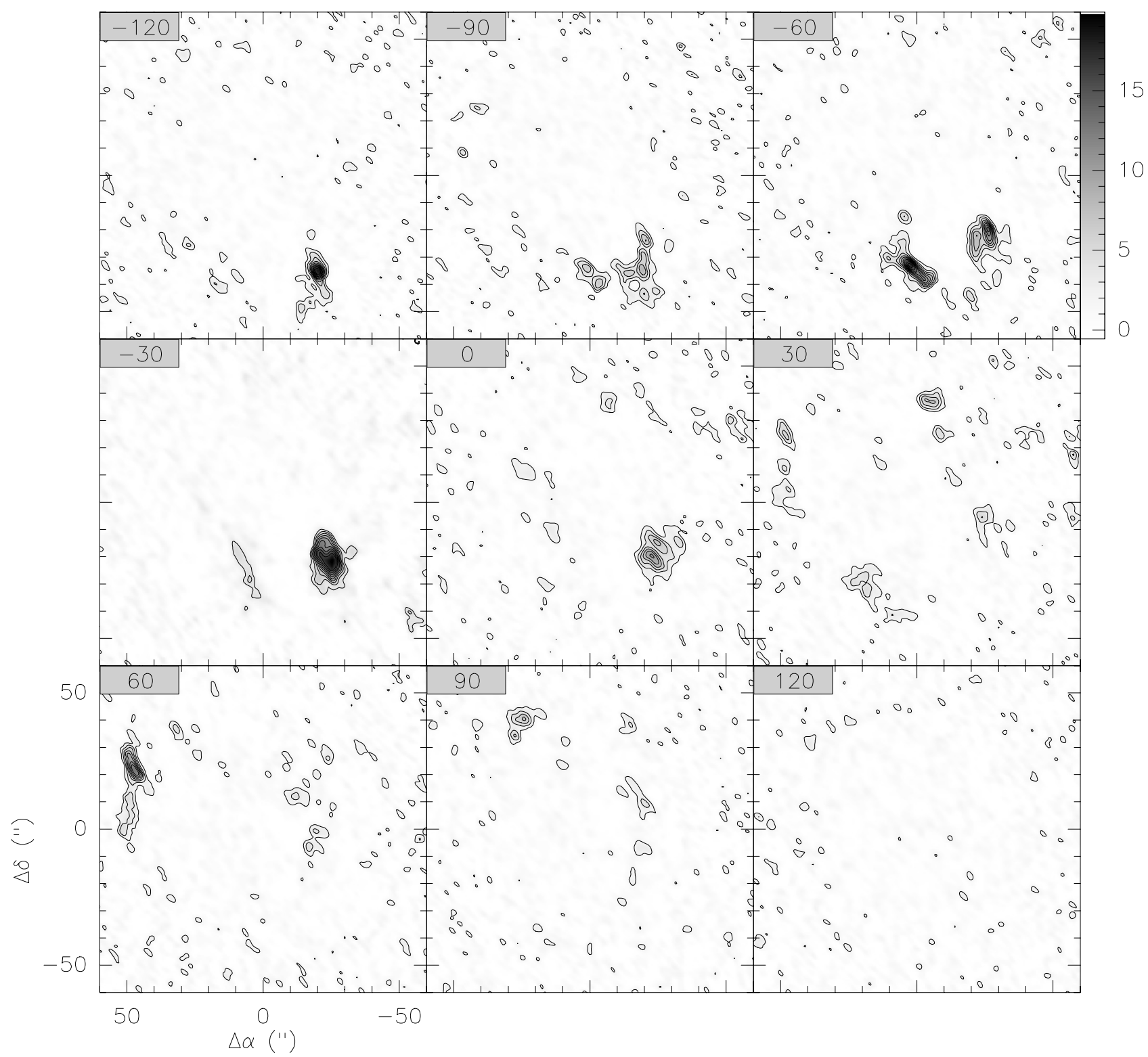

Fig. 14. Integrated intensity maps of $\mathrm{SiO} 5-4$ in velocity bins of $30 \mathrm{~km} \mathrm{~s}^{-1}$. Labels and contour steps as in Fig. 12. 


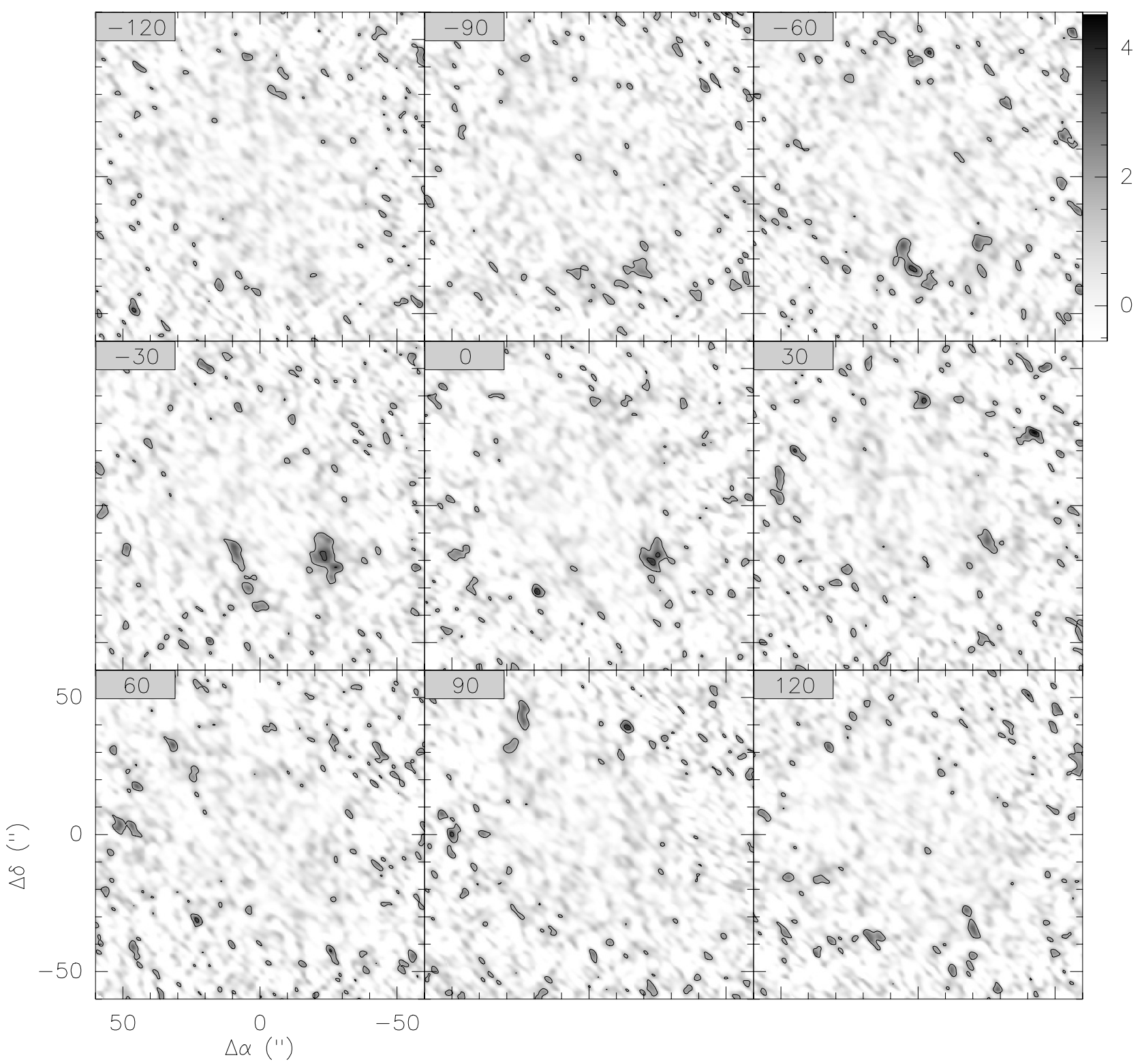

Fig. 15. Integrated intensity maps of ${ }^{13} \mathrm{CN} 2-1$ in velocity bins of $30 \mathrm{~km} \mathrm{~s}^{-1}$. Labels and contour steps as in Fig. 12. 
S. Martín et al.: Surviving the hole. I.

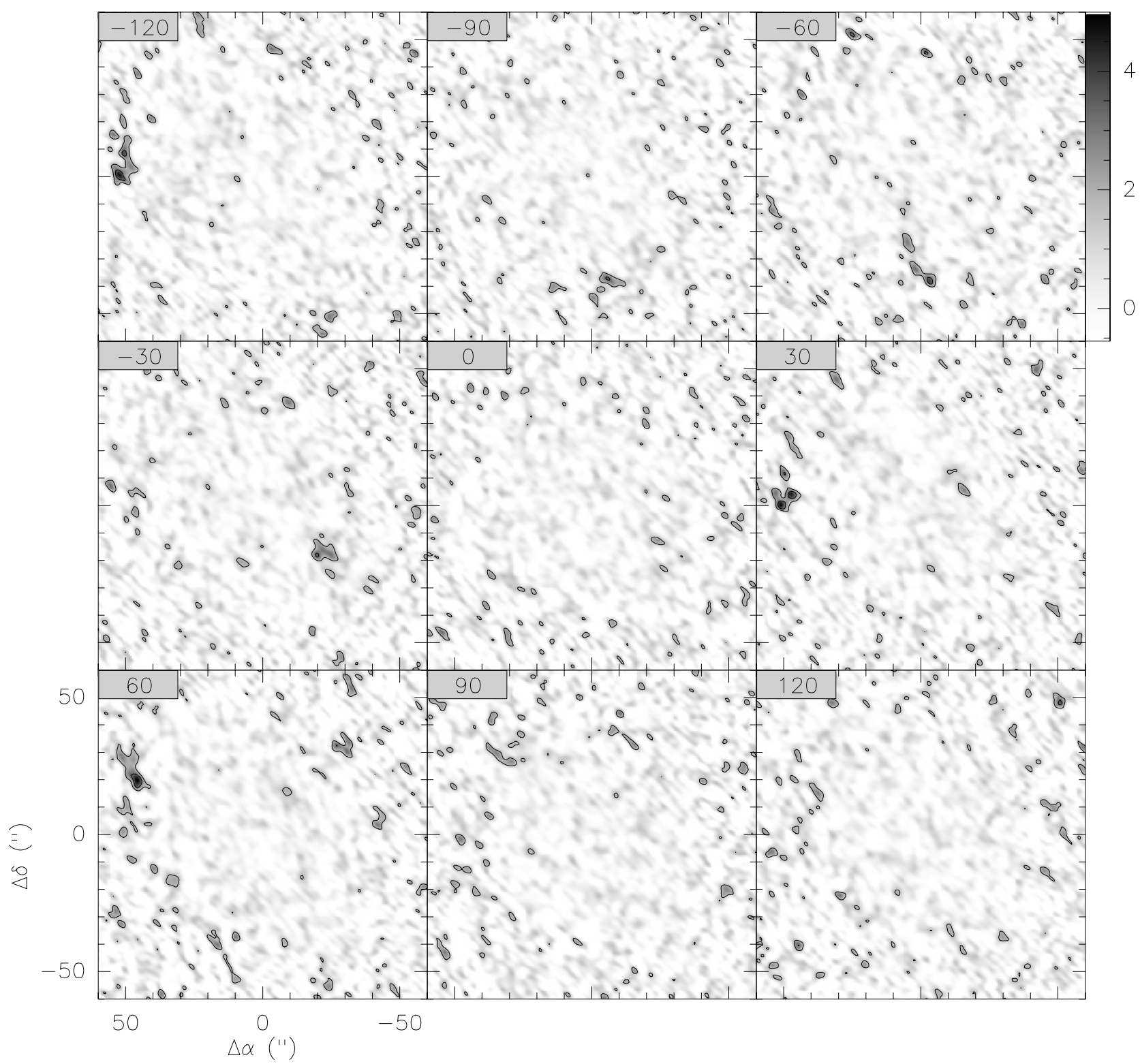

Fig. 16. Integrated intensity maps of $\mathrm{c}-\mathrm{C}_{3} \mathrm{H}_{2} 6_{1,6}-5_{0,5}$ in velocity bins of $30 \mathrm{~km} \mathrm{~s}^{-1}$. Labels and contour steps as in Fig. 12. Note that the observed emission toward the east in the $-120 \mathrm{~km} \mathrm{~s}^{-1}$ map correspond to the high-velocity emission of the $5_{1,4}-4_{2,3}$ transition of c- $\mathrm{C}_{3} \mathrm{H}_{2}(217.940 \mathrm{GHz}$, see Sect. 3). 


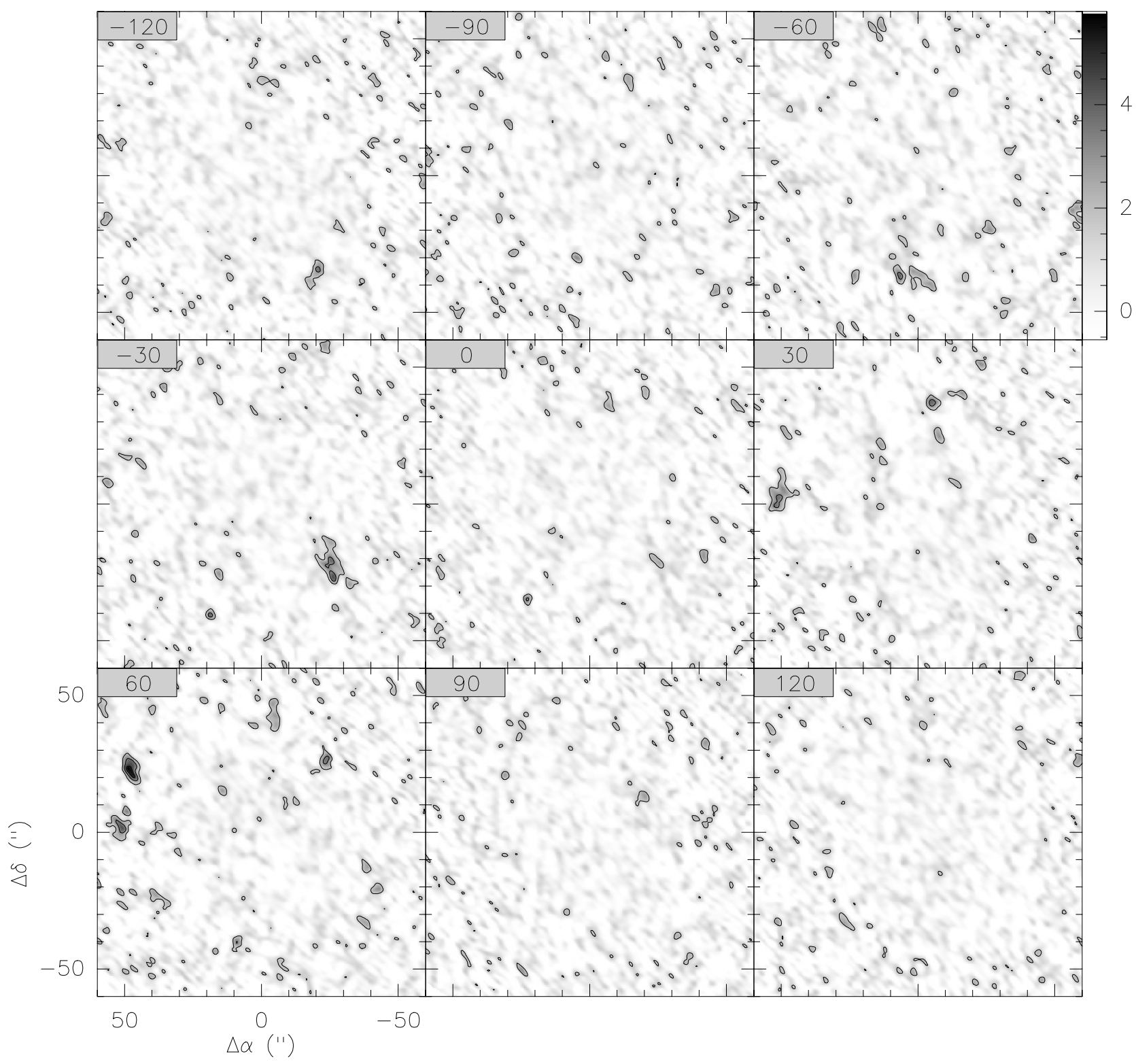

Fig. 17. Integrated intensity maps $\mathrm{HC}_{3} \mathrm{~N}$ 25-24. Labels and contour steps as in Fig. 12. 
S. Martín et al.: Surviving the hole. I.

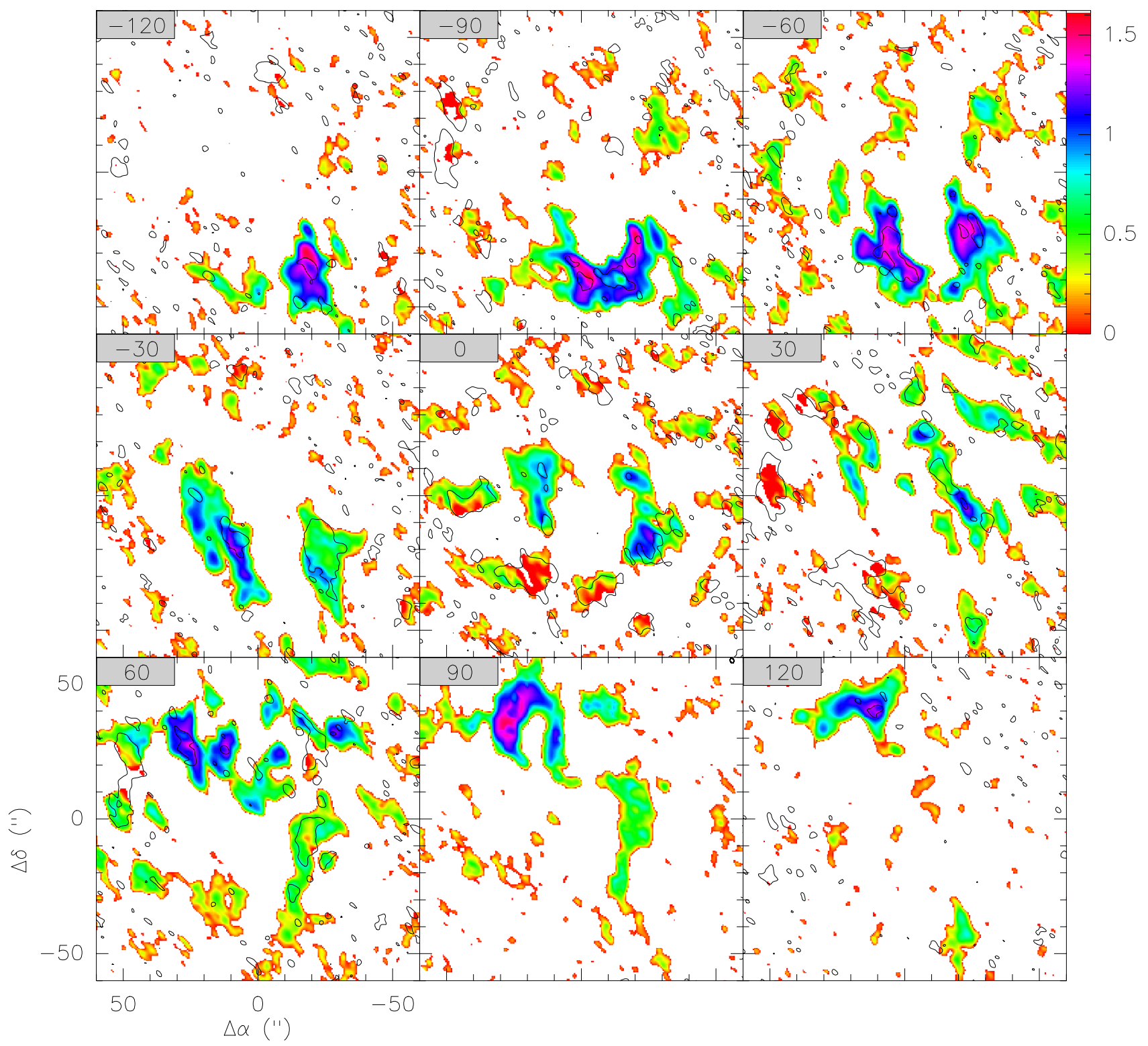

Fig. 18. Integrated $\mathrm{CN} / \mathrm{H}_{2} \mathrm{CO}$ line ratio in $30 \mathrm{~km} \mathrm{~s}^{-1}$ velocity bin maps. Color scale is logarithmic. Ratio has been computed in all pixels where $\mathrm{CN}$ emission is detected above $3 \sigma$. The contours indicate the regions where $\mathrm{H}_{2} \mathrm{CO}$ has been detected $(>3 \sigma)$. Therefore, values outside these contours represent lower limits to the line ratio where a constant $3 \sigma$ value has been assumed as the $\mathrm{H}_{2} \mathrm{CO}$ upper detection limit. 


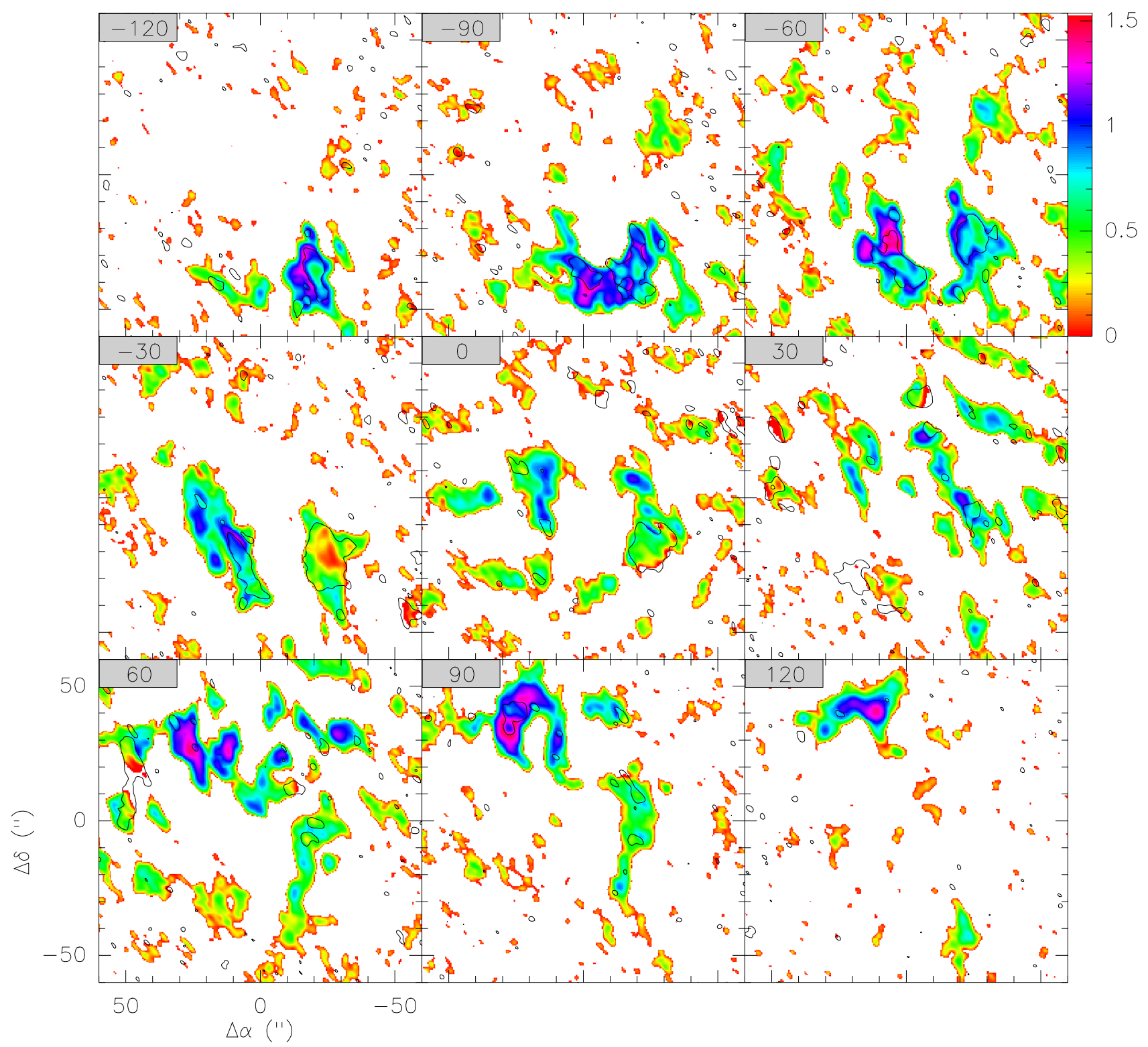

Fig. 19. Integrated $\mathrm{CN} / \mathrm{SiO}$ line ratio in $30 \mathrm{~km} \mathrm{~s}^{-1}$ velocity bin maps. Ratio has been calculated as in Fig. 18 where the contour enclose the regions where $\mathrm{SiO}$ is detected above a $>3 \sigma$ level. 
S. Martín et al.: Surviving the hole. I.

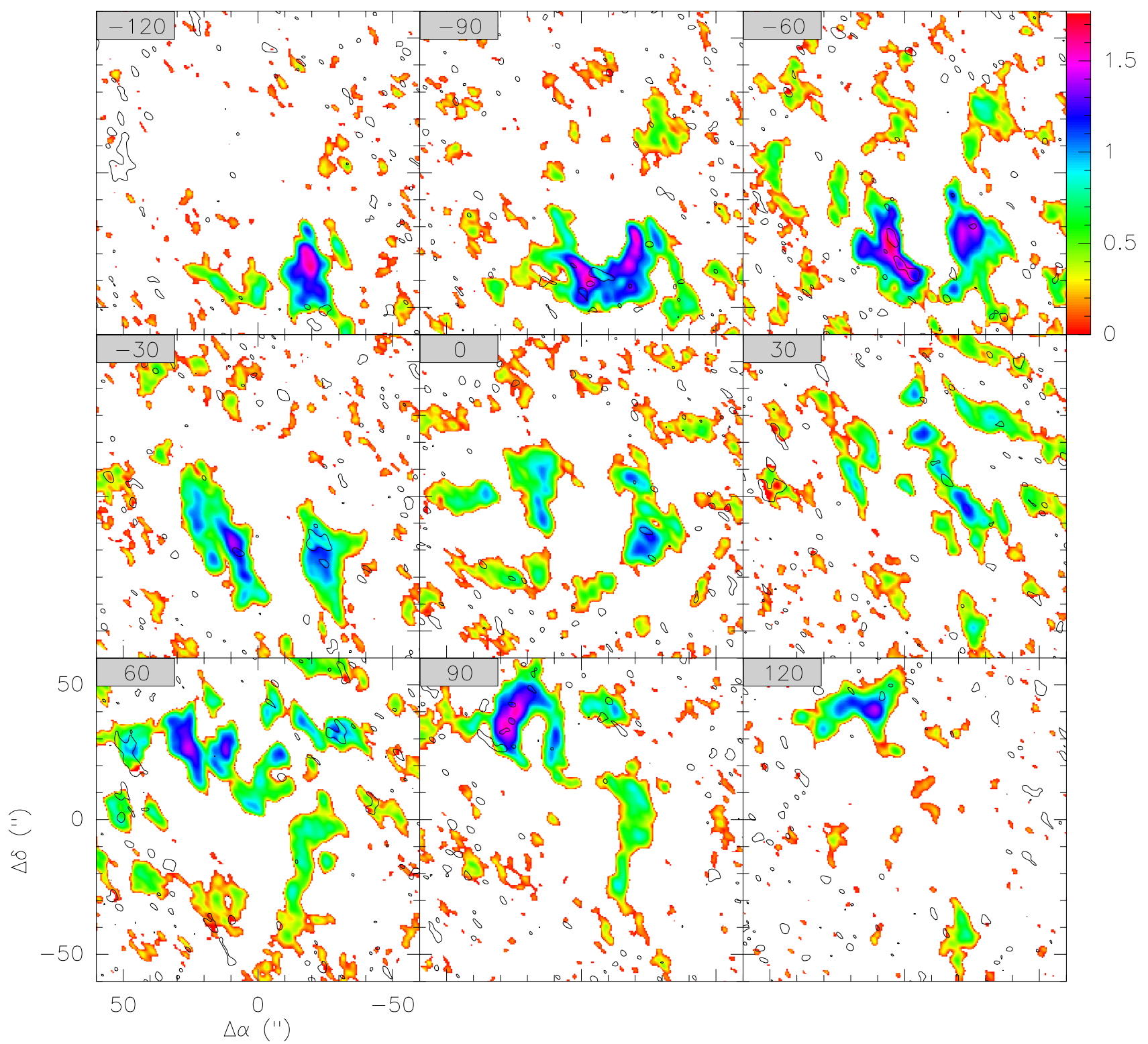

Fig. 20. Integrated $\mathrm{CN} / \mathrm{c}-\mathrm{C}_{3} \mathrm{H}_{2}$ line ratio in $30 \mathrm{~km} \mathrm{~s}^{-1}$ velocity bin maps. Ratio has been calculated as in Fig. 18 where the contour enclose the regions where $\mathrm{c}-\mathrm{C}_{3} \mathrm{H}_{2}$ is detected above a $>3 \sigma$ level. 


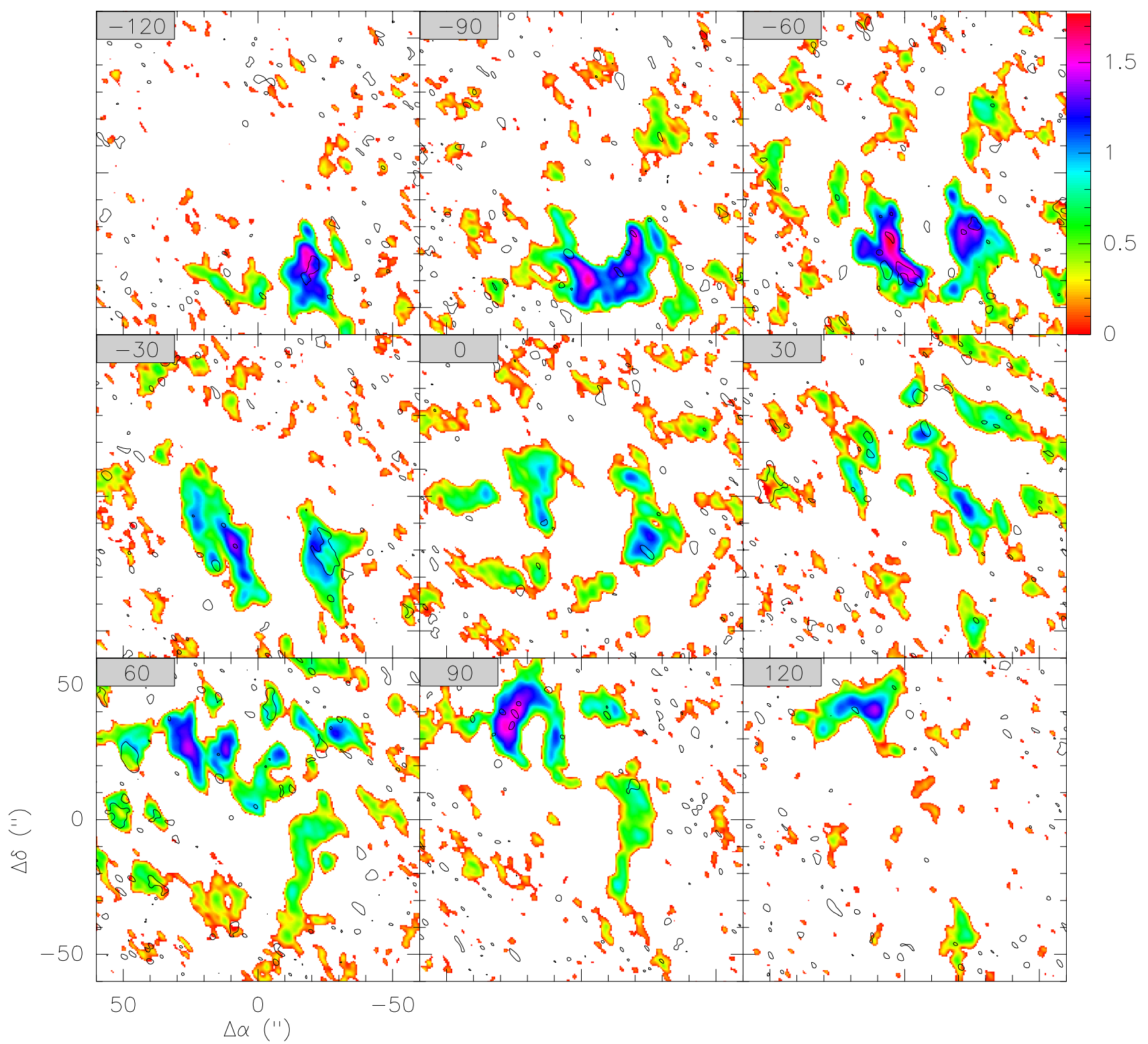

Fig. 21. Integrated $\mathrm{CN} / \mathrm{HC}_{3} \mathrm{~N}$ line ratio in $30 \mathrm{~km} \mathrm{~s}^{-1}$ velocity bin maps. Ratio has been calculated as in Fig. 18 where the contour enclose the regions where $\mathrm{HC}_{3} \mathrm{~N}$ is detected above a $>3 \sigma$ level. 
S. Martín et al.: Surviving the hole. I.

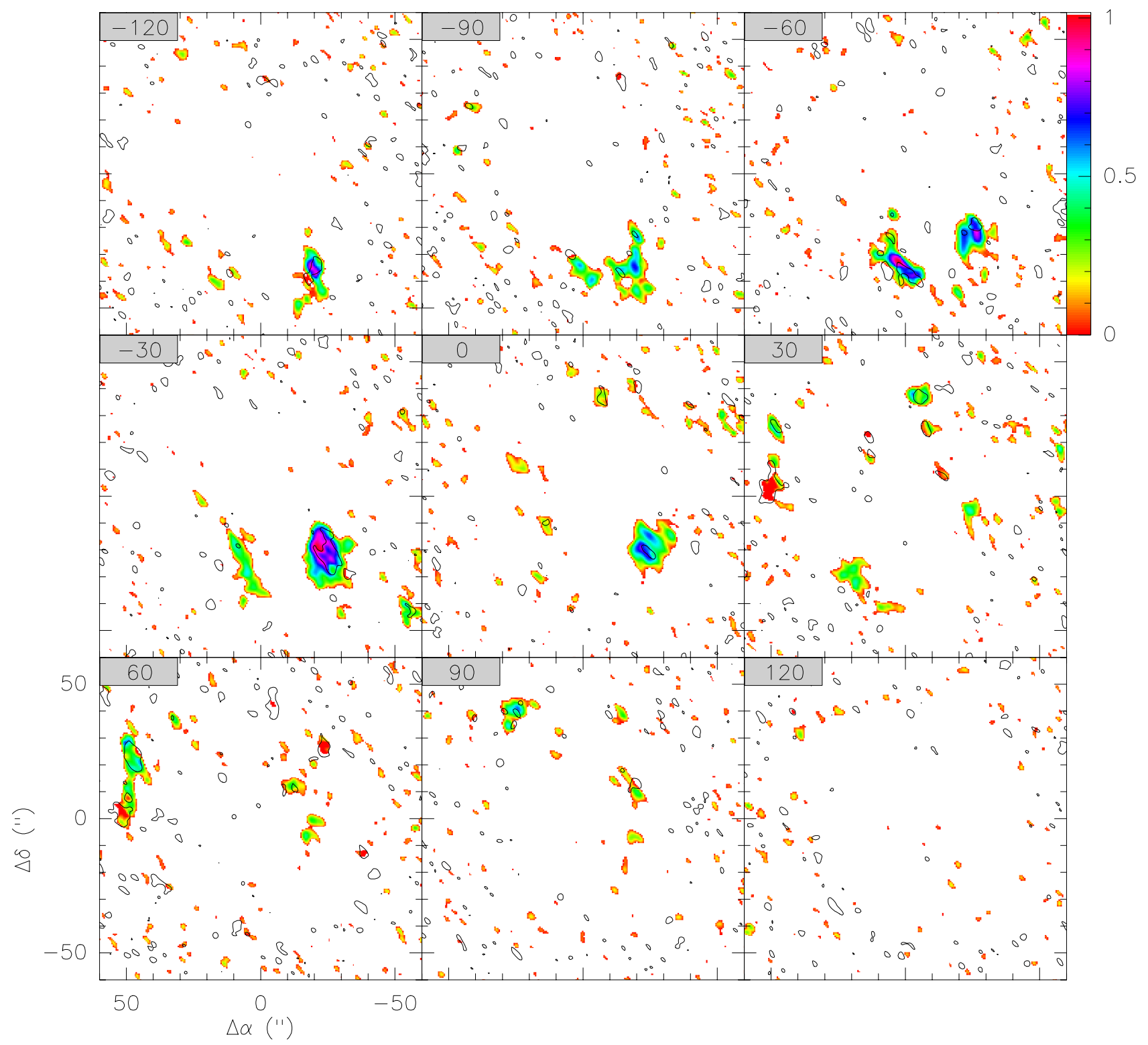

Fig. 22. Integrated $\mathrm{SiO} / \mathrm{HC}_{3} \mathrm{~N}$ line ratio in $30 \mathrm{~km} \mathrm{~s}^{-1}$ velocity bin maps. Ratio has been calculated in all pixels where $\mathrm{SiO}$ emission is detected above $3 \sigma$. Similar to Fig. 18 , the contour enclose the regions where $\mathrm{HC}_{3} \mathrm{~N}$ is detected above a $>3 \sigma$ level. 
Table 3. $\mathrm{CN}$ and ${ }^{13} \mathrm{CN}$ Gaussian fit parameters on selected positions.

\begin{tabular}{|c|c|c|c|c|c|c|c|c|c|c|}
\hline \multirow[t]{2}{*}{ Pos. } & \multirow{2}{*}{$\begin{array}{l}\Delta \alpha, \Delta \delta^{a} \\
\left({ }^{\prime \prime},{ }^{\prime \prime}\right)\end{array}$} & \multicolumn{4}{|c|}{$\mathrm{CN}$} & \multicolumn{3}{|c|}{${ }^{13} \mathrm{CN}$} & \multirow[t]{2}{*}{${ }^{12} \mathrm{CN} /{ }^{13} \mathrm{CN}^{b}$} & \multirow[t]{2}{*}{${ }^{12} \mathrm{C} /{ }^{13} \mathrm{C}^{c}$} \\
\hline & & $\underset{\left(\mathrm{Jy} \mathrm{km} \mathrm{s}{ }^{-1}\right)}{\int S \delta v}$ & $\begin{array}{c}v_{\mathrm{LSR}} \\
\mathrm{km} \mathrm{s}^{-1}\end{array}$ & $\begin{array}{c}\Delta v \\
\mathrm{~km} \mathrm{~s}^{-1}\end{array}$ & $\tau^{d}$ & $\underset{\left(\mathrm{Jy} \mathrm{km} \mathrm{s}^{-1}\right)}{\int S \delta v}$ & $\begin{array}{c}v_{\mathrm{LSR}} \\
\mathrm{km} \mathrm{s}^{-1}\end{array}$ & $\begin{array}{c}\Delta v \\
\mathrm{~km} \mathrm{~s}^{-1}\end{array}$ & & \\
\hline 1 & $(-26,-19)$ & $200 \pm 30$ & $-47.7 \pm 1.2$ & $59 \pm 3$ & $3.5 \pm 0.8$ & $9.3 \pm 1.8$ & $-30 \pm 3$ & $42 \pm 6$ & $21 \pm 5$ & 26 \\
\hline 2 & $(-20,-22)$ & $\begin{array}{c}250 \pm 17 \\
8 \pm 2\end{array}$ & $\begin{array}{r}-68.1 \pm 0.7 \\
41.6 \pm 1.8\end{array}$ & $\begin{array}{c}73.2 \pm 1.8 \\
17 \pm 4\end{array}$ & $\begin{array}{l}2.8 \pm 0.3 \\
0.1 \pm 2.0\end{array}$ & $\begin{array}{r}11 \pm 6 \\
\ldots\end{array}$ & $\begin{array}{c}-46 \pm 6 \\
\ldots\end{array}$ & $\begin{array}{c}61 \pm 16 \\
\ldots\end{array}$ & $\begin{array}{r}23 \pm 12 \\
\ldots\end{array}$ & 27 \\
\hline 3 & $(-20,-30)$ & $\begin{array}{c}120 \pm 110 \\
90 \pm 60\end{array}$ & $\begin{array}{r}-116 \pm 10 \\
-66 \pm 10\end{array}$ & $\begin{array}{l}40 \pm 30 \\
50 \pm 30\end{array}$ & $\begin{array}{l}0.5 \pm 0.1 \\
1.4 \pm 0.1\end{array}$ & $\begin{array}{c}5 \pm 2 \\
2.2 \pm 1.4\end{array}$ & $\begin{array}{l}-106 \pm 7 \\
-39 \pm 0.0\end{array}$ & $\begin{array}{l}45 \pm 16 \\
30 \pm 13\end{array}$ & $\begin{array}{l}23 \pm 23 \\
40 \pm 37\end{array}$ & $\begin{array}{l}23 \\
43\end{array}$ \\
\hline 4 & $(-20,-35)$ & $198 \pm 15$ & $-120.3 \pm 0.4$ & $46.1 \pm 1.4$ & $1.0 \pm 0.3$ & $8 \pm 2$ & $-118 \pm 5$ & $40 \pm 9$ & $26 \pm 8$ & 28 \\
\hline 5 & $(-19,-42)$ & $170 \pm 11$ & $-111.6 \pm 0.6$ & $64.8 \pm 1.6$ & $2.4 \pm 0.3$ & $4.2 \pm 1.5$ & $-89 \pm 14$ & $60 \pm 0.0$ & $40 \pm 15$ & 47 \\
\hline 6 & $(-2,-38)$ & $\begin{array}{c}200 \pm 160 \\
8 \pm 15\end{array}$ & $\begin{array}{r}-79 \pm 10 \\
-9 \pm 10\end{array}$ & $\begin{array}{l}40 \pm 30 \\
20 \pm 30\end{array}$ & $\begin{array}{l}0.3 \pm 0.1 \\
0.1 \pm 0.1\end{array}$ & $\begin{array}{c}8.5 \pm 1.6 \\
\ldots\end{array}$ & $\begin{array}{c}-72 \pm 5 \\
\ldots\end{array}$ & $\begin{array}{c}40 \pm 0.0 \\
\ldots\end{array}$ & $\begin{array}{r}24 \pm 20 \\
\ldots\end{array}$ & 23 \\
\hline 7 & $(1,-34)$ & $214 \pm 2$ & $-74.6 \pm 0.2$ & $36.9 \pm 0.3$ & $2.32 \pm 0.04$ & $12 \pm 3$ & $-72 \pm 4$ & $52 \pm 11$ & $19 \pm 5$ & 21 \\
\hline 8 & $(5,-25)$ & $181.8 \pm 1.8$ & $-60.92 \pm 0.04$ & $18.14 \pm 0.15$ & $1.72 \pm 0.02$ & $7.6 \pm 0.7$ & $-61.5 \pm 1.1$ & $18 \pm 0.0$ & $24 \pm 2$ & 26 \\
\hline \multirow[t]{2}{*}{9} & $(15,-28)$ & $\begin{array}{c}65 \pm 5 \\
12.0 \pm 1.3\end{array}$ & $\begin{array}{r}-62.6 \pm 0.2 \\
-3.4 \pm 0.6\end{array}$ & $\begin{array}{l}21.8 \pm 0.9 \\
16.8 \pm 1.4\end{array}$ & $\begin{array}{l}0.9 \pm 0.3 \\
0.1 \pm 0.2\end{array}$ & $\ldots$ & $\begin{array}{l}\ldots \\
\ldots\end{array}$ & $\begin{array}{l}\ldots \\
\ldots\end{array}$ & $\ldots$ & \\
\hline & & $13 \pm 0.0$ & $52 \pm 2$ & $24 \pm 4$ & $4 \pm 2$ & $3 \pm 3$ & $\dddot{2} \pm 10$ & $22 \pm 14$ & $4 \pm 4$ & 6 \\
\hline \multirow[t]{2}{*}{10} & $(-23,-5)$ & $57 \pm 7$ & $19.3 \pm 0.5$ & $31.0 \pm 1.7$ & $0.8 \pm 0.5$ & $3 \pm 3$ & $36 \pm 19$ & $50 \pm 30$ & $21 \pm 22$ & 22 \\
\hline & & $23 \pm 5$ & $71.6 \pm 1.0$ & $263 \pm 2$ & $0.4 \pm 0.7$ & $\ldots$ & $\ldots$ & $\ldots$ & $\ldots$ & \\
\hline \multirow[t]{2}{*}{11} & $(-24,5)$ & $17 \pm 3$ & $-4.6 \pm 0.8$ & $20 \pm 2$ & $0.1 \pm 0.7$ & $\ldots$ & $\ldots$ & $\ldots$ & $\ldots$ & \\
\hline & & $20 \pm 3$ & $68.3 \pm 0.2$ & $17 \pm 2$ & $0.1 \pm 0.3$ & $\ldots$ & $\ldots$ & $\ldots$ & $\ldots$ & \\
\hline \multirow[t]{2}{*}{12} & $(15,-2)$ & $26 \pm 7$ & $-36.1 \pm 1.5$ & $26 \pm 3$ & $4.8 \pm 1.7$ & $\ldots$ & $\ldots$ & $\ldots$ & $\ldots$ & \\
\hline & & $32 \pm 3$ & $4.3 \pm 0.4$ & $16.65 \pm 0.15$ & $1.9 \pm 0.5$ & $\ldots$ & $\ldots$ & $\ldots$ & $\ldots$ & \\
\hline 13 & $(9,-17)$ & $60 \pm 9$ & $-64.3 \pm 1.7$ & $45 \pm 3$ & $2.3 \pm 0.6$ & $\ldots$ & $\ldots$ & $\ldots$ & $\ldots$ & \\
\hline & & $69 \pm 5$ & $-39.1 \pm 0.2$ & $16.65 \pm 0.05$ & $2.4 \pm 0.3$ & $6.2 \pm 1.7$ & $-41 \pm 2$ & $25 \pm 5$ & $11 \pm 3$ & 13 \\
\hline 14 & $(25,-7)$ & $37 \pm 9$ & $-42.3 \pm 1.2$ & $30 \pm 3$ & $0.9 \pm 0.9$ & $\ldots$ & $\ldots$ & $\ldots$ & $\ldots$ & \\
\hline & & $6.8 \pm 1.8$ & $121.1 \pm 0.7$ & $32.1 \pm 1.1$ & $0.10 \pm 0.03$ & $\ldots$ & $\ldots$ & $\ldots$ & $\ldots$ & \\
\hline 15 & $(12,13)$ & $57 \pm 10$ & $11.6 \pm 0.9$ & $24.5 \pm 1.8$ & $7.3 \pm 1.6$ & $\ldots$ & $\ldots$ & $\ldots$ & $\ldots$ & \\
\hline 16 & $(21,11)$ & $20 \pm 18$ & $-12 \pm 10$ & $36 \pm 30$ & $0.1 \pm 0.1$ & $2.7 \pm 0.6$ & $33 \pm 3$ & $19.5 \pm 0.6$ & $7 \pm 7$ & 9 \\
\hline & & $26 \pm 51$ & $36 \pm 10$ & $17 \pm 30$ & $0.1 \pm 0.1$ & $\ldots$ & $\ldots$ & ... & & \\
\hline 17 & $(-8,24)$ & $69 \pm 5$ & $39.2 \pm 0.2$ & $25.3 \pm 0.6$ & $1.1 \pm 0.2$ & $3.7 \pm 0.9$ & $39 \pm 0.0$ & $25 \pm 0.0$ & $19 \pm 5$ & 20 \\
\hline 18 & $(1,5)$ & $3.8 \pm 1.0$ & $-77 \pm 2$ & $16.6 \pm 1.6$ & $0.1 \pm 0.8$ & $\ldots$ & $\ldots$ & $\ldots$ & $\ldots$ & \\
\hline & & $35 \pm 6$ & $45.8 \pm 0.6$ & $19.0 \pm 1.3$ & $2.4 \pm 0.8$ & $\ldots$ & $\ldots$ & $\ldots$ & $\ldots$ & \\
\hline 19 & $(0,0)$ & $\ldots$ & $\ldots$ & $\ldots$ & $\ldots$ & $\ldots$ & $\ldots$ & $\ldots$ & $\ldots$ & \\
\hline 20 & $(11,27)$ & $10 \pm 3$ & $-68 \pm 4$ & $31 \pm 6$ & $10 \pm 4$ & $\ldots$ & $\ldots$ & $\ldots$ & $\ldots$ & \\
\hline & & $54 \pm 2$ & $60.3 \pm 0.4$ & $22.3 \pm 0.7$ & $0.10 \pm 0.05$ & $\ldots$ & $\ldots$ & $\ldots$ & $\ldots$ & \\
\hline & & $17 \pm 0.0$ & $89.2 \pm 1.3$ & $24 \pm 2$ & $0.1 \pm 0.1$ & $\ldots$ & $\ldots$ & $\ldots$ & $\ldots$ & \\
\hline 21 & $(12,41)$ & $101 \pm 16$ & $113.6 \pm 0.7$ & $28 \pm 2$ & $2.6 \pm 0.7$ & $4 \pm 2$ & $116 \pm 4$ & $21 \pm 10$ & $26 \pm 17$ & 31 \\
\hline 22 & $(25,39)$ & $151 \pm 8$ & $86.9 \pm 0.2$ & $29.0 \pm 0.7$ & $2.0 \pm 0.2$ & $5.4 \pm 1.0$ & $90 \pm 4$ & $29 \pm 0.0$ & $28 \pm 5$ & 32 \\
\hline 23 & $(28,34)$ & $160 \pm 8$ & $80.0 \pm 0.2$ & $39.6 \pm 0.8$ & $0.78 \pm 0.18$ & $6 \pm 2$ & $76 \pm 6$ & $37 \pm 8$ & $27 \pm 9$ & 28 \\
\hline 24 & $(-3,40)$ & $8 \pm 10$ & $-84 \pm 10$ & $30 \pm 30$ & $2.3 \pm 0.1$ & $\ldots$ & $\ldots$ & $\ldots$ & $\ldots$ & \\
\hline & & $24 \pm 30$ & $31 \pm 10$ & $30 \pm 30$ & $0.3 \pm 0.1$ & $4.9 \pm 1.2$ & $31 \pm 0.0$ & $25 \pm 0.0$ & $5 \pm 7$ & 5 \\
\hline & & $25 \pm 0.0$ & $70 \pm 10$ & $30 \pm 30$ & $0.1 \pm 0.1$ & $\ldots$ & $\ldots$ & $\ldots$ & $\ldots$ & \\
\hline 25 & $(-15,40)$ & $40 \pm 10$ & $69.9 \pm 1.4$ & $318 \pm 4$ & $4.5 \pm 1.5$ & $3.7 \pm 1.2$ & $70 \pm 0.0$ & $31 \pm 0.0$ & $11 \pm 5$ & 15 \\
\hline 26 & $(-21,33)$ & $42 \pm 10$ & $38.7 \pm 0.8$ & $21 \pm 2$ & $4.0 \pm 1.2$ & $\ldots$ & $\ldots$ & $\ldots$ & $\ldots$ & \\
\hline 27 & $(-29,31)$ & $7.0 \pm 0.7$ & $-79.5 \pm 0.9$ & $16.6 \pm 0.7$ & $0.1 \pm 0.5$ & $\ldots$ & $\ldots$ & $\ldots$ & $\ldots$ & \\
\hline & & $55.3 \pm 1.8$ & $44.4 \pm 0.2$ & $16.65 \pm 0.07$ & $2.1 \pm 0.2$ & $\ldots$ & $\ldots$ & $\ldots$ & $\ldots$ & \\
\hline 28 & $(-3,45)$ & $13 \pm 2$ & $80.7 \pm 1.1$ & $20 \pm 3$ & $0.1 \pm 2$ & $\begin{array}{l}\cdots \\
\ldots\end{array}$ & $\begin{array}{l}\cdots \\
\ldots\end{array}$ & $\begin{array}{l}\cdots \\
\cdots\end{array}$ & $\begin{array}{l}\cdots \\
\ldots\end{array}$ & \\
\hline 29 & $(7,46)$ & $6 \pm 3$ & $-37 \pm 3$ & $16 \pm 5$ & $0.1 \pm 0.0$ & $\cdots$ & $\cdots$ & $\cdots$ & $\begin{array}{l}\cdots \\
\cdots\end{array}$ & \\
\hline & & $21 \pm 4$ & $108.1 \pm 1.1$ & $17 \pm 2$ & $0.1 \pm 0.0$ & $\cdots$ & $\begin{array}{l}\cdots \\
\ldots\end{array}$ & $\begin{array}{l}\cdots \\
\ldots\end{array}$ & $\begin{array}{l}\cdots \\
\ldots\end{array}$ & \\
\hline 30 & $(48,24)$ & $29.8 \pm 1.7$ & $49.1 \pm 0.4$ & $16.6 \pm 0.6$ & $0.1 \pm 0.0$ & $\cdots$ & $\ldots$ & $\cdots$ & $\cdots$ & \\
\hline 31 & $(46,20)$ & $15.9 \pm 1.1$ & $48.5 \pm 0.7$ & $16.66 \pm 0.02$ & $0.2 \pm 0.0$ & $3 \pm 2$ & $48 \pm 0.0$ & $17 \pm 0.0$ & $5 \pm 3$ & 5 \\
\hline 32 & $(50,9)$ & $14 \pm 2$ & $-56.4 \pm 1.7$ & $38 \pm 4$ & $0.42 \pm 0.15$ & $\ldots$ & $\ldots$ & $\ldots$ & $\ldots$ & \\
\hline & & $17.5 \pm 0.7$ & $38.8 \pm 0.4$ & $16.6 \pm 0.1$ & $0.145 \pm 0.002$ & $5.1 \pm 1.2$ & $35.0 \pm 1.8$ & $17 \pm 2$ & $3 \pm 0.8$ & 3 \\
\hline 33 & $(51,1)$ & $6.6 \pm 1.3$ & $-60.1 \pm 1.3$ & $16 \pm 2$ & $0.1 \pm 0.5$ & $\ldots$ & $\ldots$ & $\ldots$ & $\ldots$ & \\
\hline & & $11 \pm 2$ & $4.1 \pm 0.9$ & $16.6 \pm 0.6$ & $1.2 \pm 1.1$ & $\ldots$ & $\ldots$ & $\ldots$ & $\ldots$ & \\
\hline & & $18 \pm 0$ & $45.9 \pm 0.5$ & $16.7 \pm 1.3$ & $0.4 \pm 0.5$ & $6 \pm 2$ & $35.9 \pm 1.6$ & $17 \pm 4$ & $3 \pm 0.9$ & 3 \\
\hline 34 & $(46,-4)$ & $10 \pm 6$ & $-51 \pm 4.0$ & $33 \pm 7$ & $2 \pm 3$ & $\ldots$ & $\ldots$ & $\ldots$ & $\ldots$ & \\
\hline & & $14.2 \pm 0.8$ & $3.3 \pm 0.6$ & $16.6 \pm 0.2$ & $0.1 \pm 0.2$ & $\begin{array}{l}\cdots \\
\ldots\end{array}$ & $\begin{array}{l}\cdots \\
\ldots\end{array}$ & $\begin{array}{l}\cdots \\
\cdots\end{array}$ & $\begin{array}{l}\cdots \\
\cdots\end{array}$ & \\
\hline 35 & $(39,-2)$ & $15.6 \pm 1.5$ & $2.8 \pm 0.8$ & $16.6 \pm 0.2$ & $0.1 \pm 0.1$ & $\ldots$ & $\ldots$ & $\ldots$ & $\ldots$ & \\
\hline 36 & $(18,-34)$ & $\ldots$ & $\ldots$ & $\ldots$ & $0.1-8.2$ & $\cdots$ & $\cdots$ & $\cdots$ & $\cdots$ & \\
\hline 37 & $(-6,-36)$ & $65 \pm 8$ & $-79.1 \pm 0.6$ & $33.4 \pm 1.7$ & $0.4 \pm 0.4$ & $4 \pm 2$ & $-85 \pm 4$ & $21 \pm 12$ & $18 \pm 13$ & 18 \\
\hline & & $6.4 \pm 1.3$ & $-6.0 \pm 1.7$ & $16.6 \pm 1.5$ & $0.1 \pm 0.5$ & $\ldots$ & $\ldots$ & $\ldots$ & $\ldots$ & \\
\hline 38 & $(-14,9)$ & $33 \pm 60$ & $27 \pm 10$ & $20 \pm 30$ & $4.7 \pm 0.1$ & $\ldots$ & $\ldots$ & $\ldots$ & $\ldots$ & \\
\hline & & $7 \pm 11$ & $86 \pm 10$ & $20 \pm 30$ & $0.1 \pm 0.1$ & $\ldots$ & $\ldots$ & $\ldots$ & $\ldots$ & \\
\hline 39 & $(-19,21)$ & $11.8 \pm 1.0$ & $67.6 \pm 0.7$ & $16.6 \pm 0.7$ & $0.1 \pm 0.4$ & $\ldots$ & $\ldots$ & $\ldots$ & $\ldots$ & \\
\hline
\end{tabular}

Notes. ${ }^{(a)}$ Offset positions are relative to the nominal center of the mosaic $\alpha_{\mathrm{J} 2000}=17^{\mathrm{h}} 45^{\mathrm{m}} 40^{\mathrm{s}}, \delta_{\mathrm{J} 2000}=-29^{\circ} 00^{\prime} 28^{\prime \prime}$. $^{(b)}$ Ratio of total integrated intensity of the $\mathrm{CN}$ and ${ }^{13} \mathrm{CN}$ spectral features. ${ }^{(c)}$ Carbon isotopic ratio estimated from the opacity corrected $\mathrm{CN} /{ }^{13} \mathrm{CN}$ integrated intensity ratio. ${ }^{(d)}$ Peak optical depth of the main hyperfine component. 
S. Martín et al.: Surviving the hole. I.

Table 4. $\mathrm{H}_{2} \mathrm{CO}, \mathrm{SiO}, \mathrm{c}-\mathrm{C}_{3} \mathrm{H}_{2}$ and $\mathrm{HC}_{3} \mathrm{~N}$ Gaussian fit parameters on selected positions.

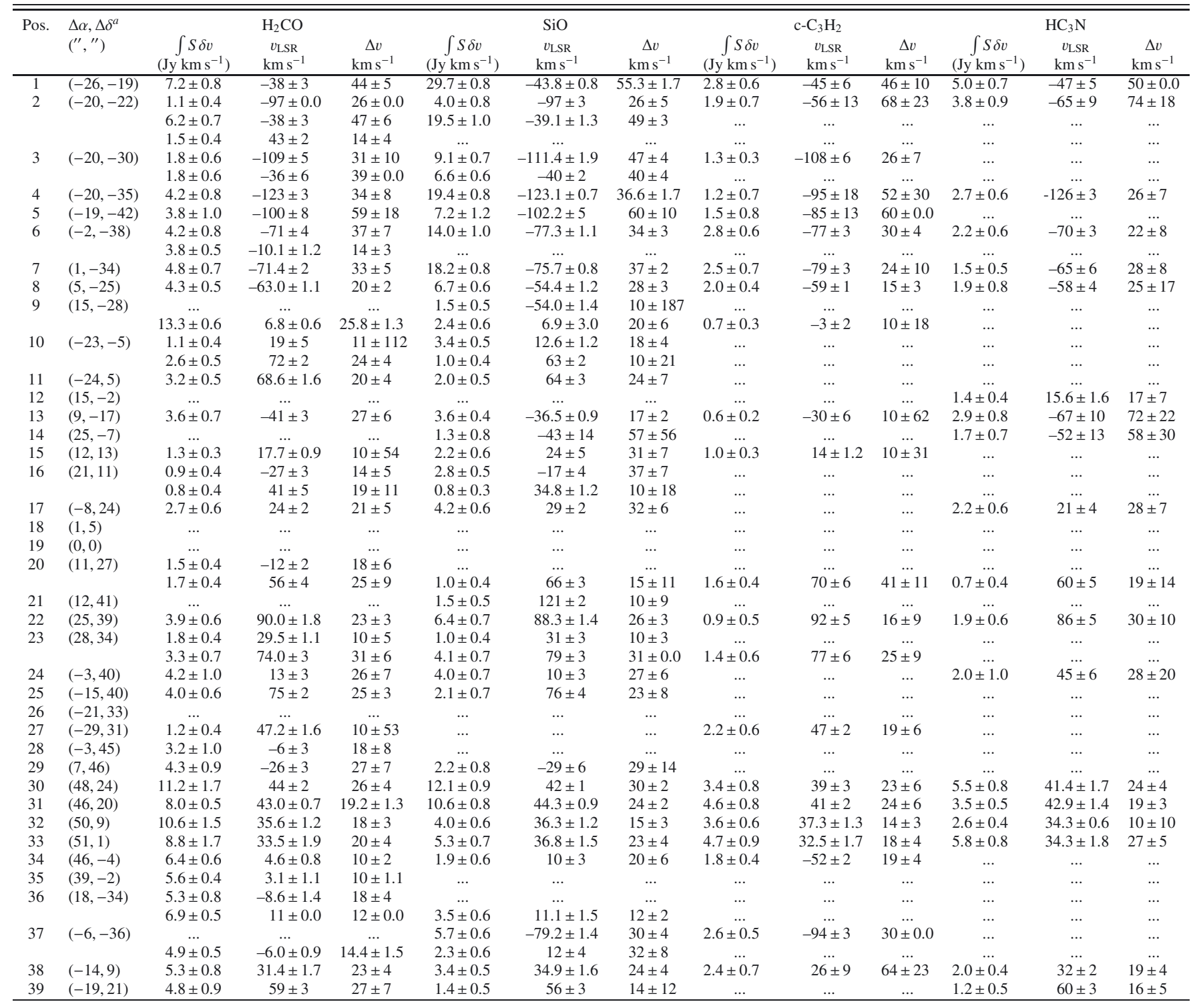

Notes. ${ }^{(a)}$ Offset positions are relative to the nominal center of the mosaic $\alpha_{\mathrm{J} 2000}=17^{\mathrm{h}} 45^{\mathrm{m}} 40^{\mathrm{s}}, \delta_{\mathrm{J} 2000}=-29^{\circ} 00^{\prime} 28^{\prime \prime}$. 